\title{
NEW HOMOGENEOUS IDEALS FOR CURRENT ALGEBRAS: FILTRATIONS, FUSION PRODUCTS AND PIERI RULES
}

\author{
GHISLAIN FOURIER
}

\begin{abstract}
New graded modules for the current algebra of $\mathfrak{s l}_{n}$ are introduced. Relating these modules to the fusion product of simple $\mathfrak{s l}_{n}$-modules and local Weyl modules of truncated current algebras shows their expected impact on several outstanding conjectures. We further generalize results on PBW filtrations of simple $\mathfrak{s l}_{n}$-modules and use them to provide decomposition formulas for these new modules in important cases.
\end{abstract}

\section{InTRODUCTION}

We consider the simple complex Lie algebra $\mathfrak{s l}_{n}=\mathfrak{b} \oplus \mathfrak{n}^{-}$and its current algebra $\mathfrak{s l}_{n} \otimes \mathbf{C}[t]$. We fix a pair $\left(\lambda_{1}, \lambda_{2}\right)$ of dominant integral $\mathfrak{s l}_{n}$-weights. $F_{\lambda_{1}, \lambda_{2}}$ will be introduced as the cyclic $\mathfrak{s l}_{n} \otimes \mathbf{C}[t]$-module defined by the homogeneous ideal generated by the kernel of an evaluation map of $\mathfrak{b} \otimes \mathbf{C}[t]$ and certain monomials in $U\left(\mathfrak{n}^{-} \otimes \mathbf{C}[t]\right) . \quad F_{\lambda_{1}, \lambda_{2}}$ decomposes into simple, finite-dimensional $\mathfrak{s l}_{n}$-modules:

$$
F_{\lambda_{1}, \lambda_{2}}={ }_{\mathfrak{s l}} \bigoplus_{\tau \in P^{+}} V(\tau)^{\oplus a_{\lambda_{1}, \lambda_{2}}^{\tau}}
$$

As $F_{\lambda_{1}, \lambda_{2}}$ is a highest weight module, we have $a_{\lambda_{1}, \lambda_{2}}^{\lambda_{1}+\lambda_{2}}=1$ and $a_{\lambda_{1}, \lambda_{2}}^{\tau}=0$ if $\tau \not \leq \lambda_{1}+\lambda_{2}$. Moreover, $\mathfrak{s l}_{n} \otimes t^{2} \mathbf{C}[t] . F_{\lambda_{1}, \lambda_{2}}=0$ and hence

$$
F_{\lambda_{1}, \lambda_{2}}=U\left(\mathfrak{n}^{-} \otimes \mathbf{C}[t] /\left(t^{2}\right)\right) \cdot \mathbb{1} \cong U\left(\mathfrak{n}^{-}\right) S\left(\mathfrak{n}^{-}\right) \cdot \mathbb{1} .
$$

Due to this observation, the $\mathfrak{s l}_{n}$-highest weight vectors and therefore the multiplicities $a_{\lambda_{1}, \lambda_{2}}^{\tau}$ should be "controlled" by $S\left(\mathfrak{n}^{-}\right)$. $\mathbb{1}$. This provides a close relation to the framework of PBW filtrations ([FFL11a, FFL13a]). By construction, $F_{\lambda_{1}, \lambda_{2}}$ is a quotient of $S\left(\mathfrak{n}^{-}\right) / \mathcal{I}\left(\lambda_{1}, \lambda_{2}\right)$ with an induced $\mathfrak{n}^{+}$-action $\circ$, where the ideal is generated by

$$
\left.U\left(\mathfrak{n}^{+}\right) \circ\left\langle f_{\alpha}^{a_{\alpha}+1}\right| \text { for all positive roots } \alpha\right\rangle \subset S\left(\mathfrak{n}^{-}\right)
$$

for some $a_{\alpha}$ depending on $\lambda_{1}, \lambda_{2}$. Generalizing the results from [FFL11a, Theorem and Theorem B] we see that a spanning set of $S\left(\mathfrak{n}^{-}\right)$. $\mathbb{1}$ can be parameterized by integer points in a polytope defined through Dyck paths conditions (Corollary 4.1). This leads to the question wether one can give a polytope parametrizing the highest weight vectors. We give a positive answer in certain important cases:

Theorem. Suppose $\lambda_{1}, \lambda_{2}$ satisfy one of the following:

(1) $\lambda_{1}, \lambda_{2}$ are both rectangular weights, e.g. multiples of some fundamental weights $\omega_{i}, \omega_{j}$,

Date: October 19, 2017.

The author was partially supported by the DFG priority program 1388 "Representation Theory". 
(2) $\lambda_{1}$ is arbitrary and $\lambda_{2}$ is either $\omega_{j}$ or $k \omega_{1}$,

(3) $\lambda_{1}+w\left(\lambda_{2}\right)$ is dominant for all Weyl group elements,

then for all dominant weights $\tau$ :

$$
a_{\lambda_{1}, \lambda_{2}}^{\tau}=c_{\lambda_{1}, \lambda_{2}}^{\tau} \text {, the Littlewood-Richardson coefficients. }
$$

Part (2) might be seen as a Pieri rule while part (3) covers $\lambda_{1} \gg \lambda_{2}$. So for fixed $\lambda_{2}$ we cover the minimal case, e.g. $\lambda_{1}$ being a fundamental weight, and the large case, e.g. $\lambda_{1} \gg \lambda_{2}$. Note that the results from [CV13, Ven13] imply $a_{\lambda_{1}, \lambda_{2}}^{\tau}=c_{\lambda_{1}, \lambda_{2}}^{\tau}$ for all $\tau \in P^{+}$if $\lambda_{1}=m \omega_{i}$ and the height of $\lambda_{2}$ is less than $m+1$. This covers of course part (1) of the theorem but we provide here a different proof using the relation to the PBW filtration.

The paper is motivated by the search for homogeneous ideals in $U\left(\mathfrak{s l}_{n} \otimes \mathbf{C}[t]\right)$ defining the fusion product $V\left(\lambda_{1}\right)_{c_{1}} * V\left(\lambda_{2}\right)_{c_{2}}$ of two simple $\mathfrak{s l}_{n}$-modules. This is the associated graded module of the tensor product of corresponding evaluation modules ([FL99] and also Section [5). These ideals can be deduced straightforward for $\mathfrak{s l}_{2}$ (Lemma 6.1) and we generalize this to obtain generators for every $\mathfrak{s l}_{2}$-triple. The theorem implies that in the considered cases (Lemma 5.1)

$$
F_{\lambda_{1}, \lambda_{2}} \cong V\left(\lambda_{1}\right)_{c_{1}} * V\left(\lambda_{2}\right)_{c_{2}}
$$

and we conjecture that this is true for all pairs of dominant integral weights.

Let us briefly explain why these modules $F_{\lambda_{1}, \lambda_{2}}$ and especially the conjectured isomorphism to the fusion product is of special interest. In fact, this is closely related to several important conjectures:

The first one is the conjecture that the fusion product of finitely many tensor factors is independent of the evaluation parameter ([FL99]). This independence has been proved for some classes of modules but so far not for arbitrary tuples of dominant integral weights. Note that two-factor case can be deduced from straightforward calculations.

The second conjecture is on Schur positivity of certain symmetric functions. In CFS14] (see also [DP07]) a partial order on pairs of dominant weights has been introduced. It is conjectured that along with the partial order, the difference of the products of the corresponding Schur functions is a non-negative linear combination of Schur functions (this has been conjectured also independently by Lam, Postnikov and Pylyavskyy), hence Schur positive. Note here, that this generalizes a conjecture on Schur positivity along row shuffles ([Oko97, FFLP05]), proved in [LPP07.

The third related conjecture is on local Weyl modules for truncated current algebras. Local Weyl modules for generalized current algebras, $\mathfrak{s l}_{n} \otimes A$, where $A$ is a commutative, associative, unital $\mathbf{C}$-algebra, have gained much attention in the last two decades. Due to their homological properties they play an important role in the category of finite-dimensional $\mathfrak{s l}_{n} \otimes A$-modules, which is not semi-simple in general (for more see [CFK10]).

Although quite a lot of research has been done on local Weyl modules, their explicit character is known for a few algebras only. For $A=\mathbf{C}\left[t^{ \pm 1}\right], \mathbf{C}[t]$ their character is given by the tensor product of fundamental modules for $\mathfrak{s l}_{n}$ (CL06, FL07]), for semi-simple, finite-dimensional $A$, the character is given by $\operatorname{dim} A$ copies of a simple $\mathfrak{s l}_{n}$-module. Besides these cases the character is not known for general local Weyl modules, not even for the "smallest" non-semi-simple algebra $A=\mathbf{C}[t] /\left(t^{2}\right)$. 
It is conjectured that for $A=\mathbf{C}[t] /\left(t^{K}\right)$ this character is also, similar to $\mathbf{C}[t]$, given by the tensor product of simple $\mathfrak{s l}_{n}$-modules. We investigate here on the $K=2$ case and prove that in this case the local Weyl modules are isomorphic to certain $F_{\lambda_{1}, \lambda_{2}}$, more detailed: $\left(\lambda_{1}, \lambda_{2}\right)$ is the unique maximal element in the aforementioned poset of pairs of dominant weights (adding up to a fixed $\lambda$ ).

A proof, that the $a_{\lambda_{1}, \lambda_{2}}^{\tau}$ are in fact the Littlewood-Richardson coefficients, would imply the conjectures on Schur positivity and on local Weyl modules immediately (Lemma 9.1, Lemma 9.2) and gives another proof for the two-factor of the independence conjecture (Lemma 5.1).

The paper is organized as follows: In Section 2 we refer to the basic definitions and in Section 3 we introduce the modules $F_{\lambda_{1}, \lambda_{2}}$, proving first properties. In Section 4 we recall the PBW filtration and work out the relation to our new modules, while in Section 5 we recall the fusion products and work out their relation to our modules. In Section 6 we give the proof for the $\mathfrak{s l}_{2}$-case and part(3) of Theorem 1. Section 7 contains the proofs of part (1) of Theorem 1 and Section 8 the proof of part (2). Section 9 recalls the partial order on pairs of dominant weights and also local Weyl modules, and relates these constructions to the new modules.

Acknowledgement: The author would like to thank Evgeny Feigin for various discussions on these modules and explaining the calculations for the independence conjecture in the twofactor case, and further Christian Korff for asking about Pieri rules.

\section{Preliminaries}

Let $\mathfrak{g}=\mathfrak{s l}_{n}(\mathbf{C})$, the special linear Lie algebra. We fix a triangular decomposition $\mathfrak{s l}_{n}=$ $\mathfrak{n}^{+} \oplus \mathfrak{h} \oplus \mathfrak{n}^{-}$and denote a fixed set of simple roots $\Pi=\left\{\alpha_{1}, \ldots, \alpha_{n-1}\right\}$, here we use the numbering from Bou02. Further, we set $I=\{i, \ldots, n-1\}$. The sets of roots is denoted R, the set of positive roots $R^{+}$. Every root $\beta \in R^{+}$can be expressed uniquely as $\alpha_{i}+\alpha_{i+1}+\ldots+\alpha_{j}$ for some $i \leq j$, we denote this root $\alpha_{i, j}$. For $\alpha \in R$, we denote the root space

$$
\left.\mathfrak{g}_{\alpha}=\left\{x \in \mathfrak{s l}_{n} \mid[h, x]=\alpha(h) x \forall h \in \mathfrak{h}\right\}=\left\langle x_{\alpha}^{+}\right| \text {if } \alpha \in R^{+}\right\rangle .
$$

Further, for $\alpha \in R^{+}$, we fix a $\mathfrak{s l}_{2}$-triple $\left\{x_{\alpha}^{+}, x_{\alpha}^{-}, h_{\alpha}\right\}$. Denote $P \subset \mathfrak{h}^{*}$, respective $P^{+}$the integral weights, respective dominant integral weights, and $\left\{\omega_{1}, \ldots, \omega_{n-1}\right\}$ the set of fundamental weights.

2.1. We recall some notations and facts from representation theory. Let $V$ be a finitedimensional $\mathfrak{s l}_{n}$-module, then $V$ decomposes into its weight spaces with respect to the $\mathfrak{h}$-action

$$
V=\bigoplus_{\tau \in P} V_{\tau}=\bigoplus_{\tau \in P}\{v \in V \mid h . v=\tau(h) . v \text { for all } h \in \mathfrak{h}\}
$$

$P^{+}$parameterizes the simple finite-dimensional modules. For $\lambda \in P^{+}$we denote the simple, finite-dimensional $\mathfrak{s l}_{n}$-module of highest weight $\lambda$ by $V(\lambda)$. Further we denote by $v_{\lambda}$ a highest weight vector of $V(\lambda)$

The category of finite-dimensional $\mathfrak{s l}_{n}$-modules is semi-simple, hence the tensor product of two simple modules decomposes into the direct sum of simple modules, so for $\lambda_{1}, \lambda_{2} \in P^{+}$

$$
V\left(\lambda_{1}\right) \otimes V\left(\lambda_{2}\right) \cong_{\mathfrak{s l}_{n}} \bigoplus_{\tau \in P^{+}} V(\tau)^{c_{\lambda_{1}, \lambda_{2}}^{\tau}}
$$


Here, $c_{\lambda_{1}, \lambda_{2}}^{\tau}$ denotes the multiplicity of the simple module $V(\tau)$ in a decomposition of the tensor product. These numbers are known as Littlewood-Richardson coefficients and there are several known formulas to compute them ([Kli68, Nak93, Lit94] to name but a few).

2.2. The vector space $\mathfrak{s l}_{n} \otimes \mathbf{C}[t]$ equipped with the bracket

$$
[x \otimes p(t), y \otimes q(t)]=[x, y] \otimes p(t) q(t) \forall x, y \in \mathfrak{s l}_{n}, p(t), q(t) \in \mathbf{C}[t]
$$

is a Lie algebra and called the current algebra of $\mathfrak{s l}_{n}$. One may also view this as the Lie algebra of regular functions on $\mathbf{C}$ with values in $\mathfrak{s l}_{n}$ (see [NSS12]). The natural grading on $\mathbf{C}[t]$ induces a grading on $U\left(\mathfrak{s l}_{n} \otimes \mathbf{C}[t]\right)$, where the component of degree 0 is $U\left(\mathfrak{s l}_{n} \otimes 1\right)$.

For a fixed $k \geq 1$, the truncated current algebra is the graded quotient of the current algebra

$$
\mathfrak{s l}_{n} \otimes \mathbf{C}[t] /\left(\mathfrak{s l}_{n} \otimes t^{K} \mathbf{C}[t]\right) \cong \mathfrak{s l}_{n} \otimes \mathbf{C}[t] /\left(t^{K}\right) .
$$

In this paper we will be dealing mainly with the $K=2$ case. Then $U\left(\mathfrak{s l}_{n} \otimes \mathbf{C}[t] /\left(t^{2}\right)\right)$ can be seen as the smash product of $U\left(\mathfrak{s l}_{n}\right)$ and the polynomial ring $S\left(\mathfrak{n}^{-}\right)$(Hag13).

2.3. The representation theory of $\mathfrak{s l}_{n} \otimes \mathbf{C}[t]$ has been subject to a lot of research during the last 25 years ([CP01, FF02, CM04, CL06, FL06, FL07, Na012] to name but a few). The most important property we should mention is, that the category of finite-dimensional $\mathfrak{s l}_{n} \otimes \mathbf{C}[t]$-modules is not semi-simple.

Every simple, finite-dimensional module is the tensor product of evaluation modules ([ER93]). This is still true if we replace $\mathbf{C}[t]$ by a commutative, finitely generated algebra $A$ and instead of complex numbers, evaluations in pairwise distinct maximal ideals [CFK10.

Although the simple, finite-dimensional modules are therefore easily described and quite well understood, the task of understanding the indecomposable modules is still unsolved (besides the case $A=\mathbf{C}[t], \mathbf{C}\left[t^{ \pm}\right]$and the cases where $A$ is finite-dimensional and semi-simple).

Even in the case where $A$ is the two-dimensional truncated polynomial ring, $A=\mathbf{C}[t] /\left(t^{2}\right)$, the category of finite-dimensional modules is far from being well understood. While the simple modules are in one-to-one correspondence to simple modules of $\mathfrak{s l}_{n}$ (by using the evaluation at the unique maximal ideal of $\mathbf{C}[t] /\left(t^{2}\right)$, CFK10] $)$, there is not much known about indecomposables, projectives etc. We will return to this point in Section 9 .

\section{Some New GRAded MOdule}

We introduce new graded modules for $\mathfrak{s l}_{n} \otimes \mathbf{C}[t]$ as follows. For fixed $\lambda_{1}, \lambda_{2} \in P^{+}$, let

$$
\left(\lambda_{1}+\lambda_{2}\right): \mathfrak{h} \longrightarrow \mathbf{C}_{\lambda_{1}+\lambda_{2}}
$$

be the one-dimensional $\mathfrak{h}$-module. We extend this trivially to an action of $\mathfrak{b}=\mathfrak{n}^{+} \oplus \mathfrak{h}$ on $\mathbf{C}_{\lambda_{1}+\lambda_{2}}$. And further, by evaluation at $t=0$, we obtain a one-dimensional module

$$
\mathfrak{b} \otimes \mathbf{C}[t] \longrightarrow \mathfrak{b} \longrightarrow \mathfrak{h} \longrightarrow \mathbf{C}_{\lambda_{1}+\lambda_{2}}
$$

We consider the induced module for the subalgebra $(\mathfrak{b} \otimes 1) \oplus\left(\mathfrak{s l}_{n} \otimes t \mathbf{C}[t]\right) \subset \mathfrak{s l}_{n} \otimes \mathbf{C}[t]$

$$
\operatorname{Ind}_{\mathfrak{b} \otimes \mathbf{C}[t]}^{\mathfrak{b} \otimes \mathfrak{s l}_{n} \otimes t \mathbf{C}[t]} \mathbf{C}_{\lambda_{1}+\lambda_{2}}
$$

and denote by $M_{\lambda_{1}, \lambda_{2}}$ the quotient by the left ideal generated by

$$
\mathfrak{n}^{-} \otimes t^{2} \mathbf{C}[t] \text { and }\left(f_{\alpha} \otimes t\right)^{\min \left\{\lambda_{1}\left(h_{\alpha}\right), \lambda_{2}\left(h_{\alpha}\right)\right\}+1}, \forall \alpha \in R^{+} .
$$


We introduce the $\mathfrak{s l}_{n} \otimes \mathbf{C}[t]$-module $F_{\lambda_{1}, \lambda_{2}}$ as the maximal integrable (as a $\mathfrak{s l}_{n}$-module) quotient

$$
F_{\lambda_{1}, \lambda_{2}}=\overline{\operatorname{Ind}_{\mathfrak{b} \otimes 1 \oplus \mathfrak{s} \mathfrak{l}_{n} \otimes t \mathbf{C}[t]}^{\mathfrak{s l}_{n} \otimes \mathbf{C}} M_{\lambda_{1}, \lambda_{2}}}
$$

Due to the construction, we can give defining relations on a generator of $F_{\lambda_{1}, \lambda_{2}}$.

Proposition 3.1. Let $\lambda_{1}, \lambda_{2} \in P^{+}$and $\lambda=\lambda_{1}+\lambda_{2}$. Then $F_{\lambda_{1}, \lambda_{2}}$ is the $\mathfrak{s l}_{n} \otimes \mathbf{C}[t]$-module generated through $w$ with relations

$$
\mathfrak{n}^{+} \otimes \mathbf{C}[t] . w=0, \mathfrak{h} \otimes t \mathbf{C}[t] . w=0, \mathfrak{n}^{-} \otimes t^{2} \mathbf{C}[t]=0
$$

and for all $\alpha \in R^{+}$and $h \in \mathfrak{h}$ :

$$
0=\left(f_{\alpha} \otimes 1\right)^{\lambda\left(h_{\alpha}\right)+1} \cdot w=\left(f_{\alpha} \otimes t\right)^{\min \left\{\lambda_{1}\left(h_{\alpha}\right), \lambda_{2}\left(h_{\alpha}\right)\right\}+1}=(h \otimes 1-\lambda(h)) . w .
$$

Proof. We have to deal with the $\mathfrak{s l}_{n}$-relation only. But since $F_{\lambda_{1}, \lambda_{2}}$ is integrable we have immediately $\left(f_{\alpha} \otimes 1\right)^{\lambda\left(h_{\alpha}\right)+1} \cdot \mathbb{1}=0$. Therefore $F_{\lambda_{1}, \lambda_{2}}$ is a quotient of the module given by the relations in the proposition. On the other hand, every module satisfying the relations is an integrable quotient of $\operatorname{Ind}_{\mathfrak{b} \otimes 1 \oplus \mathfrak{s l}_{n} \otimes t \mathbf{C}[t]}^{\mathfrak{s l}_{n} \otimes \mathbf{C}[t]} M_{\lambda_{1}, \lambda_{2}}$.

Proposition 3.2. Let $\lambda_{1}, \lambda_{2} \in P^{+}$. Then

(1) $F_{\lambda_{1}, \lambda_{2}}$ is a non-negatively graded $\mathfrak{s l}_{n} \otimes \mathbf{C}[t]$-module.

(2) $F_{\lambda_{1}, \lambda_{2}}$ is finite-dimensional.

(3) $F_{\lambda_{1}, \lambda_{2}}=\bigoplus_{s \geq 0} F_{\lambda_{1}, \lambda_{2}}^{s}$, and $F_{\lambda_{1}, \lambda_{2}}^{s}$ is a $\mathfrak{s l}_{n}$-module.

(4) $F_{\lambda_{1}, \lambda_{2}}$ has a unique simple quotient isomorphic to $V\left(\lambda_{1}+\lambda_{2}\right)_{0}$.

(5) $\mathfrak{s l}_{n} \otimes t^{2} \mathbf{C}[t] \cdot F_{\lambda_{1}, \lambda_{2}}=0$, and hence $F_{\lambda_{1}, \lambda_{2}}$ is a $\mathfrak{s l}_{n} \otimes \mathbf{C}[t] /\left(t^{2}\right)$-module.

Proof. Part (1) is clear, since the defining relations of $F_{\lambda_{1}, \lambda_{2}}$ are homogeneous and $U\left(\mathfrak{s l} \mathfrak{l}_{n} \otimes \mathbf{C}[t]\right)$ is non-negatively graded. Due to the defining relations, $F_{\lambda_{1}, \lambda_{2}}$ is a quotient of the local graded Weyl module for $U\left(\mathfrak{s l}_{n} \otimes \mathbf{C}[t]\right)$ of highest weight $\lambda_{1}+\lambda_{2}, W_{\mathbf{C}[t]}\left(0, \lambda_{1}+\lambda_{2}\right)$ (see Proposition 9.1 or [CP01] for details, they are not relevant here). In [CP01 it is shown that this local graded Weyl module is finite-dimensional, which implies (2).

Now, as $\mathfrak{s l}_{n} \cong \mathfrak{s l}_{n} \otimes 1 \hookrightarrow \mathfrak{s l}_{n} \otimes \mathbf{C}[t], F_{\lambda_{1}, \lambda_{2}}$ is also a finite-dimensional $\mathfrak{s l}_{n}$-module, hence decomposes into a direct sum of simple finite-dimensional $\mathfrak{s l}_{n}$-modules. Moreover, as $U\left(\mathfrak{s l}_{n}\right)$ is the degree 0 part of $U\left(\mathfrak{s l}_{n} \otimes \mathbf{C}[t]\right)$, we see that each graded component $F_{\lambda_{1}, \lambda_{2}}^{s}$ is a $\mathfrak{s l}_{n}$-module and each simple $\mathfrak{s l}_{n}$-module is contained in a unique $F_{\lambda_{1}, \lambda_{2}}^{s}$. This implies (3).

The degree 0 component of $F_{\lambda_{1}, \lambda_{2}}$ is obviously isomorphic to $V\left(\lambda_{1}+\lambda_{2}\right)$ as a $\mathfrak{s l}_{n}$-module. A standard argument shows that

$$
U\left(\mathfrak{s l}_{n} \otimes \mathbf{C}[t]\right) \mathfrak{s l}_{n} \otimes t \mathbf{C}[t] . \mathbb{1}
$$

is the maximal proper submodule not containing $\mathbb{1}$. The quotient by this submodule is isomorphic to the graded evaluation module $V\left(\lambda_{1}+\lambda_{2}\right)_{0}$, this gives (4). Part (5) follows again immediately from the defining relations.

Since $F_{\lambda_{1}, \lambda_{2}}^{s}$ is a $\mathfrak{s l}_{n}$-module, it has a decomposition into a direct sum of simple $\mathfrak{s l}_{n}$-modules

$$
F_{\lambda_{1}, \lambda_{2}}^{s} \cong_{\mathfrak{s l}_{n}} \bigoplus_{\tau \in P^{+}} V(\tau)^{\oplus a_{\lambda_{1}, \lambda_{2}}^{\tau}(s)} \text {, for some } a_{\lambda_{1}, \lambda_{2}}^{\tau}(s) \geq 0 .
$$


We set

$$
a_{\lambda_{1}, \lambda_{2}}^{\tau}:=\sum_{s \geq 0} a_{\lambda_{1}, \lambda_{2}}^{\tau}(s)
$$

so we have

$$
\operatorname{dim} \operatorname{Hom}_{\mathfrak{s l}_{n}}\left(F_{\lambda_{1}, \lambda_{2}}, V(\tau)\right)=a_{\lambda_{1}, \lambda_{2}}^{\tau} .
$$

We see immediately from Proposition 3.1

Corollary 3.1. Let $\lambda_{1}, \lambda_{2} \in P^{+}$, then

$$
a_{\lambda_{1}, \lambda_{2}}^{\lambda_{1}+\lambda_{2}}=1 ; a_{\lambda_{1}, \lambda_{2}}^{\tau}=0 \text { for } \tau \not \leq \lambda_{1}+\lambda_{2} \text {. }
$$

The main theorem of the paper is the following:

Theorem 3.1. Let $\lambda_{1}, \lambda_{2} \in P^{+}$, then we have $a_{\lambda_{1}, \lambda_{2}}^{\tau} \geq c_{\lambda_{1}, \lambda_{2}}^{\tau}, \forall \tau \in P^{+}$.

Moreover:

(1) (Pieri rules) Let $\lambda_{1} \in P^{+}, \lambda_{2} \in\left\{\omega_{j}, k \omega_{1}\right\}$ for some $j \in I$ or $k \geq 1$, then: $a_{\lambda_{1}, \lambda_{2}}^{\tau}=c_{\lambda_{1}, \lambda_{2}}^{\tau}$, $\forall \tau \in P^{+}$.

(2) Let $\lambda_{1}=m_{i} \omega_{i}, \lambda=m_{j} \omega_{j}$ for some $i, j \in I, m_{i}, m_{j} \geq 0$, then: $a_{\lambda_{1}, \lambda_{2}}^{\tau}=c_{\lambda_{1}, \lambda_{2}}^{\tau}, \forall \tau \in P^{+}$.

(3) If $\lambda_{1} \gg \lambda_{2}$, then: $a_{\lambda_{1}, \lambda_{2}}^{\tau}=c_{\lambda_{1}, \lambda_{2}}^{\tau}, \forall \tau \in P^{+}$.

The proofs will be given in the following sections, but we should note the following here:

Remark 3.1. In the proof we will see that $\lambda_{1} \gg \lambda_{2}$ can be made precise, by requesting

$$
c_{\lambda_{1}, \lambda_{2}}^{\tau}=\operatorname{dim} V\left(\lambda_{2}\right)_{\tau-\lambda_{1}}
$$

for all $\tau \in P^{+}$. Note that this is equivalent to $\lambda_{1}+w\left(\lambda_{2}\right) \in P^{+}$for all $w \in W$, the Weyl group of $\mathfrak{s l}_{n}$.

Remark 3.2. From the work [CV13, Ven13] one can deduce further that $a_{\lambda_{1}, \lambda_{2}}^{\tau}=c_{\lambda_{1}, \lambda_{2}}^{\tau}$ if $\lambda_{1}=m \omega_{i}$ and $\lambda_{2}\left(h_{\theta}\right) \leq m$ (where $\theta$ is the highest root of $\mathfrak{s l}_{n}$ ). The authors were using relations on Demazure modules and their fusion products, generalizing an approach presented in [FL06. This of course includes (2) of the theorem but we give a new proof here that might be generalized to other but rectangular weights.

\section{PBW FiLtration AND POLYTOPES}

In this section we recall the PBW filtration and we will see how the results from [FFL11a] can be adapted here in order to understand the $\mathfrak{s l}_{n}$-structure on $F_{\lambda_{1}, \lambda_{2}}$.

By the PBW theorem and the construction of $F_{\lambda_{1}, \lambda_{2}}$ as an induced module we know that

$$
F_{\lambda_{1}, \lambda_{2}}=U\left(\mathfrak{n}^{-}\right) U\left(\mathfrak{n}^{-} \otimes t\right) . \mathbb{1} .
$$

In order to understand the $\mathfrak{s l}_{n}$-decomposition of $F_{\lambda_{1}, \lambda_{2}}$ it would be sufficient to parametrize all $\mathfrak{s l}_{n}$-highest weight vectors. The equation above suggests, that this set of highest weight vectors should be controlled by $U\left(\mathfrak{n}^{-} \otimes t\right) . \mathbb{1}$. We start with analyzing this. 
4.1. We recall the notion of Dyck path from [FFL11a]:

A Dyck path of length $s$ is a sequence of positive roots

$$
\mathbf{p}=\left(\beta_{1}, \ldots, \beta_{s}\right)
$$

with $s \geq 1$ and such that if $\beta_{i}=\alpha_{k, \ell}$ then $\beta_{i+1} \in\left\{\alpha_{k+1, \ell}, \alpha_{k, \ell+1}\right\}$. If $\beta_{1}=\alpha_{k_{1}, \ell_{1}}$ and $\beta_{s}=\alpha_{k_{s}, \ell_{s}}$, then we call

$$
\alpha_{k_{1}, \ell_{s}} \text { the base root of the path } \mathbf{p} \text {, denoted by } \beta(\mathbf{p})
$$

Denote the set of all Dyck paths by $\mathbb{D}$.

4.2. The PBW filtration on $U\left(\mathfrak{n}^{-}\right)$is given as follows:

$$
\left.U\left(\mathfrak{n}^{-}\right)^{\leq s}=\left\langle x_{1} \cdots x_{r}\right| 0 \leq r \leq s \text { and } x_{i} \in \mathfrak{n}^{-}\right\rangle_{\mathbf{C}}
$$

The associated graded algebra is a commutative algebra isomorphic to $S\left(\mathfrak{n}^{-}\right)$, the polynomial ring in $\mathfrak{n}^{-}$. The adjoint action of $\mathfrak{n}^{+}$on $\mathfrak{s l}_{n}$ induces an action $\circ$ on $S\left(\mathfrak{n}^{-}\right)$.

We fix a tuple of non-negative integers

$$
\mathbf{a}:=\left(a_{\alpha}\right) \in \mathbb{Z}_{\geq 0}^{n(n-1) / 2}
$$

and consider the ideal $\mathcal{I}(\mathbf{a}) \subset S\left(\mathfrak{n}^{-}\right)$given by

$$
\mathcal{I}(\mathbf{a})=S\left(\mathfrak{n}^{-}\right)\left\langle\sum_{\alpha \in R^{+}} U\left(\mathfrak{n}^{+}\right) \circ f_{\alpha}^{a_{\alpha}+1}\right\rangle .
$$

4.3. We fix $\mathbf{a}=\left(a_{\alpha}\right)$ and define a polytop in $\mathbb{R}^{n(n-1) / 2}$ :

$$
\mathcal{P}(\mathbf{a})=\left\{\left(x_{\alpha}\right) \in \mathbb{R}^{n(n-1) / 2} \mid \forall \mathbf{p} \in \mathbb{D}: \sum_{\alpha \in \mathbf{p}} x_{\alpha} \leq a_{\beta(\mathbf{p})}\right\} .
$$

We denote

$$
S(\mathbf{a})=\mathcal{P}(\mathbf{a}) \cap \mathbb{Z}_{\geq 0}^{n(n-1) / 2}
$$

the set of integer points in $\mathcal{P}(\mathbf{a})$.

This construction of the polytope covers the cases considered in FFL11a, FFL11b, FFL13b, FFL13a, Gor11, BD14, where $a_{\alpha}:=\lambda\left(h_{\alpha}\right)$ for some fixed $\lambda \in P^{+}$.

We define further the degree and the weight of an integer point: Let $\mathbf{s}=\left(s_{\alpha}\right) \in \mathbb{Z}_{\geq 0}^{n(n-1) / 2}$, then

$$
\operatorname{deg}(\mathbf{s})=\sum_{\alpha \in R^{+}} s_{\alpha} \text { and } \operatorname{wt}(\mathbf{s})=\sum_{\alpha \in R^{+}} s_{\alpha} \alpha \in P
$$

4.4. Although, our approach generalizes the construction provided in [FFL11a, we obtain a similar result on a spanning set of $S\left(\mathfrak{n}^{-}\right) / \mathcal{I}(\mathbf{a})$ (see [FFL11a, Theorem 2]). For this denote

$$
\mathbf{f}^{\mathbf{t}}=\prod_{\alpha \in R^{+}} f_{\alpha}^{t_{\alpha}} \in S\left(\mathfrak{n}^{-}\right) \text {where } \mathbf{t}=\left(t_{\alpha}\right) \in \mathbb{Z}_{\geq 0}^{n(n-1) / 2} .
$$


Lemma 4.1. We fix $\mathbf{a}=\left(a_{\alpha}\right) \in \mathbb{Z}_{\geq 0}^{n(n-1) / 2}$, then

$$
\left\{\overline{\mathbf{f}^{\mathbf{s}}} \mid \mathbf{s} \in S(\mathbf{a})\right\}
$$

is a spanning set of $S\left(\mathfrak{n}^{-}\right) / \mathcal{I}(\mathbf{a})$.

Proof. Here we follow the idea in [FFL11a]. $\mathfrak{n}^{+}$acts by differential operators on $S\left(\mathfrak{n}^{-}\right)$, namely $e_{\alpha} \circ f_{\beta}=f_{\beta-\alpha}$ or 0 if $\beta-\alpha$ is a positive root. Using these differential operators and an appropriate total order $\prec$ on the monomials in $S\left(\mathfrak{n}^{-}\right)$, we can prove in exactly the same way as [FFL11a, Proposition 1] a straightening law. Namely if $\mathbf{s} \notin S(\mathbf{a})$, then

$$
\overline{\mathbf{f}^{\mathrm{s}}}=\overline{\sum_{\mathbf{t} \prec \mathbf{s}} c_{\mathrm{t}} \mathbf{f}^{\mathrm{t}}} .
$$

This implies now the lemma. For more details we refer to [FFL11a].

In FFL11a, and the case $a_{\alpha}:=\lambda\left(h_{\alpha}\right)$, for some fixed $\lambda \in P^{+}$, it was further proved that this set is in fact a basis. We can not prove this here and although we conjecture that this is also true in our generality.

4.5. By construction $M_{\lambda_{1}, \lambda_{2}}$ is a cyclic $U\left(\mathfrak{n}^{-} \otimes t\right)$-module. So there exists an ideal $\mathcal{I}_{\lambda_{1}, \lambda_{2}}$ such that

$$
M_{\lambda_{1}, \lambda_{2}} \cong U\left(\mathfrak{n}^{-} \otimes t\right) / \mathcal{I}_{\lambda_{1}, \lambda_{2}} .
$$

Since $M_{\lambda_{1}, \lambda_{2}}$ is a $\mathfrak{n}^{+}$-module, the ideal $\mathcal{I}_{\lambda_{1}, \lambda_{2}}$ is stable under the adjoint action of $\mathfrak{n}^{+}$(on $\left.U\left(\mathfrak{n}^{-} \otimes t\right)\right)$. Moreover the action is a graded action (where $\mathfrak{n}^{+}$has degree 0 ). Note that we have the identification

$$
S\left(\mathfrak{n}^{-}\right) \cong U\left(\mathfrak{n}^{-} \otimes t\right) \subset U\left(\mathfrak{s l}_{n} \otimes \mathbf{C}[t] /\left(t^{2}\right)\right) \text { via } \mathbf{f}^{\mathbf{t}} \mapsto \prod_{\alpha}\left(f_{\alpha} \otimes t\right)^{t_{\alpha}} .
$$

Then we have the obvious proposition:

Proposition 4.1. For $\lambda_{1}, \lambda_{2} \in P^{+}$we set

$$
\mathbf{a}:=\left(a_{\alpha}\right) \text { where } a_{\alpha}=\min \left\{\lambda_{1}\left(h_{\alpha}\right), \lambda_{2}\left(h_{\alpha}\right)\right\} .
$$

Then we have maps of $S\left(\mathfrak{n}^{-}\right)$-modules

$$
S\left(\mathfrak{n}^{-}\right) / \mathcal{I}(\mathbf{a}) \rightarrow M_{\lambda_{1}, \lambda_{2}} \rightarrow U\left(\mathfrak{n}^{-} \otimes t\right) . \mathbb{1} \subset F_{\lambda_{1}, \lambda_{2}} .
$$

To emphasize the dependence on $\lambda_{1}, \lambda_{2}$, we denote the set of integer points $S(\mathbf{a})$ in this case by $S\left(\lambda_{1}, \lambda_{2}\right)$. Then, combining Proposition 4.1 and Lemma 4.1, we have:

Corollary 4.1. Fix $\lambda_{1}, \lambda_{2} \in P^{+}$, then

$$
\left\{\mathbf{f}^{\mathbf{s}} . \mathbb{1} \mid \mathbf{s} \in S\left(\lambda_{1}, \lambda_{2}\right)\right\}
$$

is, via the identification, a spanning set for $M_{\lambda_{1}, \lambda_{2}}$ and hence for $U\left(\mathfrak{n}^{-} \otimes t\right) . \mathbb{1} \subset F_{\lambda_{1}, \lambda_{2}}$. 
4.6. In order to identify the $\mathfrak{s l}_{n}$-highest weight vectors in $F_{\lambda_{1}, \lambda_{2}}$ with images of $\prod_{\alpha}(f \otimes$ $t)^{s_{\alpha}}$. $\mathbb{1}$ for some $\mathbf{s} \in S\left(\lambda_{1}, \lambda_{2}\right)$, we introduce an appropriate filtration of $U\left(\mathfrak{n}^{-} \otimes t\right)$. First we filter by the degree of $t$ and the further by the height of the weights. Finally, we filter further by a total order on the monomials.

Recall that $U\left(\mathfrak{n}^{-} \otimes t\right) \cong S\left(\mathfrak{n}^{-}\right)$if considered as the subalgebra in $U\left(\mathfrak{s l}_{n} \otimes \mathbf{C}[t] / t^{2}\right)$ as we continue to do. Therefore $U\left(\mathfrak{n}^{-} \otimes t\right)$ is naturally graded by $t$ and we keep denoting the graded components $U\left(\mathfrak{n}^{-} \otimes t\right)^{s}$. For $\tau \in P$, we denote

$$
U\left(\mathfrak{n}^{-} \otimes t\right)_{\tau}=\left\{v \in U\left(\mathfrak{n}^{-} \otimes t\right) \mid \operatorname{wt}(v)=\tau\right\} .
$$

All weights of $U\left(\mathfrak{n}^{-} \otimes t\right)$ are in $\bigoplus_{i \in I} \mathbb{Z}_{\leq 0} \alpha_{i}$. Let $\tau=\sum_{i \in I} a_{i} \alpha_{I} \in \bigoplus_{i \in I} \mathbb{Z}_{\leq 0} \alpha_{i}$. Then we denote the height of $\tau$

$$
\operatorname{ht}(\tau):=\sum_{i \in I}-a_{i}
$$

So we have a filtration of the graded components

$$
\left.U\left(\mathfrak{n}^{-} \otimes t\right)^{s, \leq \ell}=\left\langle u \in U\left(\mathfrak{n}^{-} \otimes t\right)_{\tau}^{s}\right| \mathrm{ht}(\tau) \leq \ell\right\} .
$$

This is spanned by monomials of total degree $s$ and whose weights have height less or equals to $\ell$.

On the other hand, $U\left(\mathfrak{n}^{-} \otimes t\right)$ is $\mathbb{Z}_{>0}^{n(n-1) / 2}$ graded. Each graded component is one-dimensional, spanned by $\prod_{\alpha \in R^{+}}\left(f_{\alpha} \otimes t\right)^{s_{\alpha}}$ for some $\mathbf{s} \in \mathbb{Z}_{\geq 0}^{n(n-1) / 2}$. We order the $n(n-1) / 2$-tuples by first ordering the positive roots

$$
\alpha_{i, j} \leq \alpha_{k, \ell}: \Leftrightarrow i<k \text { or } i=k \text { and } j \leq \ell .
$$

Using the lexicographic order $\leq$ we obtain an order on the monomials spanning $U\left(\mathfrak{n}^{-} \otimes t\right)$.

Combining this we introduce a finer filtration on $U\left(\mathfrak{n}^{-} \otimes t\right)^{s}$. So given $s, \ell \geq 0$ and $\mathbf{n} \in \mathbb{Z}_{\geq 0}^{n(n-1) / 2}$ with $\operatorname{deg}(\mathbf{n})=s, \operatorname{ht}(-\operatorname{wt}(\mathbf{n}))=\ell$, we have

$U\left(\mathfrak{n}^{-} \otimes t\right)^{s, \leq \ell} \underset{\mathbf{n}}{s, \leq \ell}=U\left(\mathfrak{n}^{-} \otimes t\right)^{s,<\ell}+\left\langle\prod_{\alpha \in R^{+}}\left(f_{\alpha} \otimes t\right)^{m_{\alpha}} \mid \operatorname{deg}(\mathbf{m})=s, \operatorname{ht}(-\operatorname{wt} \mathbf{m})=\ell, \mathbf{m}<\mathbf{n}\right\rangle_{\mathbf{C}}$.

4.7. We turn back to the module $F_{\lambda_{1}, \lambda_{2}}$ and recall its graded components $F_{\lambda_{1}, \lambda_{2}}^{s}$. We define

$$
\mathcal{F}^{\leq \ell}\left(F_{\lambda_{1}, \lambda_{2}}^{s}\right):=U\left(\mathfrak{s l}_{n}\right) U\left(\mathfrak{n}^{-} \otimes t\right)^{s, \leq \ell} . \mathbb{1} \subseteq F_{\lambda_{1}, \lambda_{2}}^{s}
$$

By construction

$$
\mathcal{F}^{\leq \ell}\left(F_{\lambda_{1}, \lambda_{2}}^{s}\right) / \mathcal{F}^{<\ell}\left(F_{\lambda_{1}, \lambda_{2}}^{s}\right)
$$

is a $\mathfrak{s l}_{n}$-module and we have the following

Lemma 4.2. Let $\mathbf{s} \in S\left(\lambda_{1}, \lambda_{2}\right)$, then the image of

$$
\mathbf{f}^{\mathbf{s}} . \mathbb{1} \in \mathcal{F}^{\leq \ell}\left(F_{\lambda_{1}, \lambda_{2}}^{s}\right) / \mathcal{F}^{<\ell}\left(F_{\lambda_{1}, \lambda_{2}}^{s}\right)
$$

is either 0 or a $\mathfrak{s l}_{n}$-highest weight vector of weight $\lambda_{1}+\lambda_{2}-$ wt(s). 
Proof. Since ht $\left(e_{\beta}\right)>0$, we see using the commutator relations that

$$
e_{\beta} \prod_{\alpha}\left(f_{\alpha} \otimes t\right)^{s_{\alpha}} \in U\left(\mathfrak{n}^{-} \otimes t\right)^{s, \leq \ell} U\left(\mathfrak{n}^{+}\right)_{+}+U\left(\mathfrak{n}^{-} \otimes t\right)^{s,<\ell} U\left(\mathfrak{n}^{+}\right) .
$$

This implies that

$$
e_{\alpha} \prod_{\alpha}\left(f_{\alpha} \otimes t\right)^{s_{\alpha}}=0 \in \mathcal{F}^{\leq \ell}\left(F_{\lambda_{1}, \lambda_{2}}^{s}\right) / \mathcal{F}^{<\ell}\left(F_{\lambda_{1}, \lambda_{2}}^{s}\right) .
$$

We see that, by choosing this appropriate filtration, the highest weight vectors (for the $\mathfrak{s l}_{n}$-action) of the associated graded module $F_{\lambda_{1}, \lambda_{2}}$, are of the form $\mathbf{f}^{\mathbf{s}}$. $\mathbb{1}$ for some $\mathbf{s}$.

By using the refinement of the filtration we can say even more. So given $s, \ell \geq 0$ and $\mathbf{n} \in$ $\mathbb{Z}_{\geq 0}^{n(n-1) / 2}$ with $\operatorname{deg}(\mathbf{n})=s, \operatorname{ht}(-\operatorname{wt}(\mathbf{n}))=\ell$, we have

$$
\mathcal{F}_{\leq \mathbf{n}}^{\leq \ell}\left(F_{\lambda_{1}, \lambda_{2}}^{s}\right):=U\left(\mathfrak{s l}_{n}\right) U\left(\mathfrak{n}^{-} \otimes t\right)_{\leq \mathbf{n}}^{s, \leq \ell} \cdot \mathbb{1} \subset F_{\lambda_{1}, \lambda_{2}}^{s} .
$$

Then the graded components

$$
\mathfrak{G}_{\mathbf{n}}^{s, \ell}\left(F_{\lambda_{1}, \lambda_{2}}\right):=\mathcal{F}_{\leq \mathbf{n}}^{\leq \ell}\left(F_{\lambda_{1}, \lambda_{2}}^{s}\right) /\left(\mathcal{F}^{<\ell}\left(F_{\lambda_{1}, \lambda_{2}}^{s}\right)+\sum_{\mathbf{m}<\mathbf{n}} \mathcal{F}_{\leq \mathbf{m}}^{\leq \ell}\left(F_{\lambda_{1}, \lambda_{2}}^{s}\right)\right)
$$

are simple $\mathfrak{s l}_{n}$-modules.

4.8. We have seen in Corollary 4.1 that the monomials corresponding to points in $S\left(\lambda_{1}, \lambda_{2}\right)$ are a spanning set of $U\left(\mathfrak{n}^{-} \otimes t\right) . \mathbb{1}$.

Definition 4.1. We say $\mathbf{n} \in S\left(\lambda_{1}, \lambda_{2}\right)$ is a highest weight point if $\mathfrak{G}_{\mathbf{n}}^{s, \ell}\left(F_{\lambda_{1}, \lambda_{2}}\right)$ is non-zero for $s=\operatorname{deg}(\mathbf{n})$ and $\ell=\operatorname{ht}(-\operatorname{wt}(\mathbf{n}))$. The set of highest weight points is denoted $S_{h w}\left(\lambda_{1}, \lambda_{2}\right)$.

Note that, since $F_{\lambda_{1}, \lambda_{2}}$ is an integrable $\mathfrak{s l}_{n}$-module, we have for all $\mathbf{s} \in S_{h w}\left(\lambda_{1}, \lambda_{2}\right)$

$$
\lambda_{1}+\lambda_{2}-\operatorname{wt}(\mathbf{s}) \in P^{+} .
$$

Corollary 4.2. For $\lambda_{1}, \lambda_{2}, \tau \in P^{+}$we have

$$
\operatorname{dim} \operatorname{Hom}_{\mathfrak{s l}_{n}}\left(F_{\lambda_{1}, \lambda_{2}}, V(\tau)\right)=\sharp\left\{\mathbf{s} \in S_{h w}\left(\lambda_{1}, \lambda_{2}\right) \mid \operatorname{wt}(\mathbf{s})=\lambda_{1}+\lambda_{2}-\tau\right\}
$$

Moreover

$$
\operatorname{dim} \operatorname{Hom}_{\mathfrak{s l}_{n}}\left(F_{\lambda_{1}, \lambda_{2}}^{s}, V(\tau)\right)=\sharp\left\{\mathbf{s} \in S_{h w}\left(\lambda_{1}, \lambda_{2}\right) \mid \operatorname{wt}(\mathbf{s})=\lambda_{1}+\lambda_{2}-\tau \text { and } \operatorname{deg}(\mathbf{s})=s .\right\}
$$

\section{FUSION PRODUCTS}

In this section we recall the fusion product of two simple $\mathfrak{s l}_{n}$-modules and work out the relation to the modules $F_{\lambda_{1}, \lambda_{2}}$. 
5.1. The following construction is due to [FL99]. Recall the grading on $U\left(\mathfrak{s l}_{n} \otimes \mathbf{C}[t]\right)$ given by the degree function on $\mathbf{C}[t]$

$$
U\left(\mathfrak{s l}_{n} \otimes \mathbf{C}[t]\right)^{r}=\left\{u \in U\left(\mathfrak{s l}_{n} \otimes \mathbf{C}[t]\right) \mid \operatorname{deg}(u) \leq r\right\} .
$$

Then $\mathcal{F}^{0}=U\left(\mathfrak{s l}_{n}\right)$ and we set $\mathcal{F}^{-1}=0$.

Let $V\left(\lambda_{1}\right), \ldots, V\left(\lambda_{k}\right)$ be simple $\mathfrak{s l}_{n}$-modules of highest weights $\lambda_{1}, \ldots, \lambda_{k}$. Further let $c_{1}, \ldots, c_{k}$ be pairwise distinct complex numbers. Then $V\left(\lambda_{i}\right)$ can be endowed with the structure of a $\mathfrak{s l}_{n} \otimes \mathbf{C}[t]$-module via

$$
x \otimes p(t) \cdot v=p\left(c_{i}\right) x \cdot v \quad \text { for all } x \in \mathfrak{s l}_{n}, p(t) \in \mathbf{C}[t], v \in V\left(\lambda_{i}\right),
$$

we denote this module $V\left(\lambda_{i}\right)_{c_{i}}$. Then

$$
V\left(\lambda_{1}\right)_{c_{1}} \otimes \cdots \otimes V\left(\lambda_{k}\right)_{c_{k}}
$$

is cyclic generated by the tensor product of highest weight vectors $v_{\lambda_{1}} \otimes \cdots \otimes v_{\lambda_{k}}$ (even more it is simple [ER93, CFK10]). The grading on $U\left(\mathfrak{s l}_{n} \otimes \mathbf{C}[t]\right)$ induces a filtration on $V\left(\lambda_{1}\right)_{c_{1}} \otimes \cdots \otimes V\left(\lambda_{k}\right)_{c_{k}}$

$$
U\left(\mathfrak{s l}_{n} \otimes \mathbf{C}[t]\right)^{\leq r} . v_{\lambda_{1}} \otimes \cdots \otimes v_{\lambda_{k}} .
$$

Since $U\left(\mathfrak{s l}_{n} \otimes \mathbf{C}[t]\right)$ is graded, the associated graded is again a module for $U\left(\mathfrak{s l}_{n} \otimes \mathbf{C}[t]\right)$, denoted usually by

$$
V\left(\lambda_{1}\right)_{c_{1}} * \cdots * V\left(\lambda_{k}\right)_{c_{k}}
$$

and is called the fusion product. Recall that the graded components are $U\left(\mathfrak{s l}_{n}\right)$-modules, since $U(\mathfrak{s l} \otimes 1)$ is the degree 0 component of $U\left(\mathfrak{s l}_{n} \otimes \mathbf{C}[t]\right)$. Further, since we have not changed the $\mathfrak{s l}_{n}$-structure in this construction:

Corollary 5.1. Let $\lambda_{1}, \lambda_{2} \in P^{+}, c_{1} \neq c_{2} \in \mathbf{C}$, then for all $\tau \in P^{+}$

$$
\operatorname{dim} \operatorname{Hom}_{\mathfrak{s l}_{n}}\left(V\left(\lambda_{1}\right)_{c_{1}} * V\left(\lambda_{2}\right)_{c_{2}}, V(\tau)\right)=c_{\lambda_{1}, \lambda_{2}}^{\tau} \text {. }
$$

5.2 .

Lemma 5.1. For $\lambda_{1}, \lambda_{2} \in P^{+}, c_{1} \neq c_{2} \in \mathbf{C}$ we have a surjective map of $\mathfrak{s l}_{n} \otimes \mathbf{C}[t]$-modules:

$$
F_{\lambda_{1}, \lambda_{2}} \rightarrow V\left(\lambda_{1}\right)_{c_{1}} * V\left(\lambda_{2}\right)_{c_{2}}
$$

moreover $a_{\lambda_{1}, \lambda_{2}}^{\tau} \geq c_{\lambda_{1}, \lambda_{2}}^{\tau}, \forall \tau \in P^{+}$.

Proof. We prove the $\mathfrak{s l}_{2}$-case first. Here dominant integral weights are parameterized by $\mathbb{Z}_{\geq 0}$, and for $k \geq 0$ let $V(k)=S y m^{k} \mathbf{C}^{2}$. Fix $k \geq m \geq 0$. Then

$$
\operatorname{dim}\left(V(k)_{c_{1}} \otimes V(m)_{c_{2}}\right)_{k+m-2 \ell}=\left\{\begin{array}{l}
\ell+1 \text { for } 0 \leq \ell \leq m \\
m+1 \text { for } m \leq \ell \leq k \\
k+m+1-\ell \text { for } k \leq \ell \leq k+m
\end{array}\right.
$$

Since $c_{1} \neq c_{2}$, we se, using the Vandermonde determinant, that

$$
\left(f_{\alpha} \otimes t\right)^{m+1} v_{k} \otimes v_{m} \in\left\langle\left(f_{\alpha} \otimes 1\right)^{m+1} v_{k} \otimes v_{m}, \ldots,\left(f_{\alpha} \otimes 1\right)\left(f_{\alpha} \otimes t\right)^{m} v_{k} \otimes v_{m}\right\rangle_{\mathbf{C}}
$$

since the $k-2$-weight space is at most $m+1$-dimensional. This implies that $\left(f_{\alpha} \otimes t\right)^{m+1} v_{k} \otimes v_{m}$ is 0 in the associated graded module.

We see further, that the weight space of weight $k+m-2$ is two dimensional and spanned by the vectors $\left(f_{\alpha} \otimes 1\right) v_{k} \otimes v_{m},\left(f_{\alpha} \otimes t\right) v_{k} \otimes v_{m}$. This implies that for $\ell \geq 2,\left(f_{\alpha} \otimes t^{\ell}\right) v_{k} \otimes v_{m}=0$ in 
the fusion product, similarly we see that for all $\ell \geq 1, h \otimes t^{\ell} v_{k} \otimes v_{m}=0$ in the fusion product. This implies that there is a surjective map of $\mathfrak{s l}_{2}$-modules

$$
F_{k \omega, m \omega} \rightarrow V(k)_{c_{1}} * V(m)_{c_{2}}
$$

Let us turn to the general case. Let $\lambda_{1}, \lambda_{2} \in P^{+}, c_{1} \neq c_{2} \in \mathbf{C}, \alpha \in R^{+}$, and let $m=$ $\min \left\{\lambda_{1}\left(h_{\alpha}\right), \lambda_{2}\left(h_{\alpha}\right)\right\}$. By considering the $\mathfrak{s l}_{2}$-triple $\left\{e_{\alpha}, h_{\alpha}, f_{\alpha}\right\}$ we see with the same argument as above that

$$
\left(f_{\alpha} \otimes t\right)^{m+1} v_{\lambda_{1}} \otimes v_{\lambda_{2}} \in \operatorname{span}\left\{\left(f_{\alpha} \otimes 1\right)^{m+1} v_{\lambda_{1}} \otimes v_{\lambda_{2}}, \ldots,\left(f_{\alpha} \otimes 1\right)\left(f_{\alpha} \otimes t\right)^{m} v_{\lambda_{1}} \otimes v_{\lambda_{2}}\right\}
$$

This implies that $\left(f_{\alpha} \otimes t\right)^{m+1} v_{\lambda_{1}} \otimes v_{\lambda_{2}}=0$ in the associated graded. The remaining defining relations for $F_{\lambda_{1}, \lambda_{2}}$ are easily verified.

Using this lemma we have the following very interesting consequence:

Corollary 5.2. If $\forall \tau \in P^{+}: a_{\lambda_{1}, \lambda_{2}}^{\tau}=c_{\lambda_{1}, \lambda_{2}}^{\tau}$, then for all $c_{1} \neq c_{2} \in \mathbf{C}$ :

$$
V\left(\lambda_{1}\right)_{c_{1}} * V\left(\lambda_{2}\right)_{c_{2}} \cong_{\mathfrak{s l}_{n} \otimes \mathbf{C}[t]} F_{\lambda_{1}, \lambda_{2}} .
$$

Moreover, the fusion product in this case is independent of the parameter $c_{1}, c_{2}$, providing another proof of a conjecture by B.Feigin and S.Loktev ([FL99]).

Proof. By Lemma 5.1 we have for all $\lambda_{1}, \lambda_{2} \in P^{+}$and $c_{1} \neq c_{2} \in \mathbf{C}$ a surjective map of $\mathfrak{s l}_{n} \otimes \mathbf{C}[t]$-modules

$$
F_{\lambda_{1}, \lambda_{2}} \rightarrow V\left(\lambda_{1}\right)_{c_{1}} * V\left(\lambda_{2}\right)_{c_{2}}
$$

With Corollary 5.1 we know that the multiplicity of $V(\tau)$ in the fusion product is $c_{\lambda_{1}, \lambda_{2}}^{\tau}$. By assumption, this is equal to $a_{\lambda_{1}, \lambda_{2}}^{\tau}$, which is the multiplicity of $V(\tau)$ in $F_{\lambda_{1}, \lambda_{2}}$. So the modules are isomorphic as $\mathfrak{s l}_{n}$-modules and hence by a dimension argument also as $\mathfrak{s l}_{n} \otimes \mathbf{C}[t]$-modules. Since $F_{\lambda_{1}, \lambda_{2}}$ is a graded module and independent of any evaluation parameter, the same is true for the fusion product $V\left(\lambda_{1}\right)_{c_{1}} * V\left(\lambda_{2}\right)_{c_{2}}$.

\section{FIRST PROOFS FOR PARTS OF THE MAIN THEOREM}

We prove here the $\mathfrak{s l}_{2}$-case, namely $a_{m \omega_{1}, k \omega_{1}}^{\tau}=c_{m \omega_{1}, k \omega_{1}}^{\tau}$ for all $m, k \geq 0$ and $\tau \in P^{+}$. In the following section we prove the $\lambda_{1} \gg \lambda_{2}$-case.

6.1. In this section we consider the $\mathfrak{s l}_{2}$-case. In this case, dominant integral weights are parametrized by non-negative integers.

Lemma 6.1. Let $m_{1}, m_{2} \geq 0$, then for all $c_{1} \neq c_{2} \in \mathbf{C}$

$$
F_{m_{1} \omega_{1}, m_{2} \omega_{1}} \cong_{\mathfrak{s l}_{2} \otimes \mathbf{C}[t]} V\left(m_{1} \omega_{1}\right)_{c_{1}} * V\left(m_{2} \omega_{1}\right)_{c_{2}} .
$$

Moreover, $a_{m_{1} \omega_{1}, m_{2} \omega_{1}}^{k \omega_{1}}=c_{m_{1} \omega_{1}, m_{2} \omega_{1}}^{k \omega_{1}}$.

This proves Theorem 3.1 (1) for $A_{1}$. 
Proof. Let $m_{1}, m_{2} \in \mathbb{Z}_{\geq 0}$, then by Lemma 5.1 it suffices to prove that $\forall k \geq 0$

$$
a_{m_{1} \omega_{1}, m_{2} \omega_{1}}^{k \omega_{1}} \leq c_{m_{1} \omega_{1}, m_{2} \omega_{1}}^{k \omega_{1}}
$$

Suppose $m_{1} \geq m_{2}$. Then the relations of $F_{m_{1} \omega_{1}, m_{2} \omega_{2}}$ can be rewritten as

$$
(h \otimes 1) \cdot \mathbb{1}=\left(m_{1}+m_{2}+1\right) \cdot \mathbb{1} ; ;(f \otimes 1)^{m_{1}+m_{2}+1} \cdot \mathbb{1}=0 ;(f \otimes t)^{m_{2}+1} \cdot \mathbb{1}=0,
$$

while $\left(\mathfrak{n}^{-} \otimes t^{2} \mathbf{C}[t] \oplus \mathfrak{b} \otimes t \mathbf{C}[t] \oplus \mathfrak{n}^{+} \otimes 1\right) . \mathbb{1}=0$. By considering $F_{m_{1} \omega_{1}, m_{2} \omega_{1}}$ as an $\mathfrak{s l}_{2}$-module we see from the relations, that it is generated by

$$
\left\{\mathbb{1},(f \otimes t) . \mathbb{1},(f \otimes t)^{2} \cdot \mathbb{1}, \ldots,,(f \otimes t)^{m_{2}} \cdot \mathbb{1}\right\} .
$$

This implies that $F_{m_{1} \omega_{1}, m_{2} \omega_{1}}$ is multiplicity free and moreover we see that

$$
a_{m_{1} \omega_{1}, m_{2} \omega_{1}}^{k \omega_{1}}=1 \Rightarrow k=m_{1}+m_{2}-2 \ell \text { for some } \ell \in\left\{0, \ldots, m_{2}\right\} .
$$

The famous Clebsch-Gordan formula gives for $k=m_{1}+m_{2}-2 \ell$ for some $\ell \in\left\{0, \ldots, m_{2}\right\}$

$$
c_{m_{1} \omega_{1}, m_{2} \omega_{1}}^{k \omega_{1}}=1 \text { and } c_{m_{1} \omega_{1}, m_{2} \omega_{1}}^{k \omega_{1}}=0 \text { else } .
$$

This implies (with Lemma 5.1)

$$
a_{m_{1} \omega_{1}, m_{2} \omega_{1}}^{k \omega_{1}} \leq c_{m_{1} \omega_{1}, m_{2} \omega_{1}}^{k \omega_{1}} \leq a_{m_{1} \omega_{1}, m_{2} \omega_{1}}^{k \omega_{1}}
$$

Note here, that this elementary result follows also from [FF02] and CV13.

6.2. Let $\lambda_{1}, \lambda_{2} \in P^{+}$. We say

$$
\lambda_{1} \gg \lambda_{2} \Leftrightarrow \lambda_{1}+w\left(\lambda_{2}\right) \in P^{+} \Leftrightarrow c_{\lambda_{1}, \lambda_{2}}^{\tau}=\operatorname{dim} V\left(\lambda_{2}\right)_{\tau-\lambda_{1}}, \forall \tau \in P^{+} .
$$

This is certainly satisfied if $\lambda_{1}\left(h_{\alpha}\right) \gg \lambda\left(h_{\alpha}\right)$ for all $\alpha \in R^{+}$.

Suppose now $\lambda_{1} \gg \lambda_{2}$, then $\min \left\{\lambda_{1}\left(h_{\alpha}\right), \lambda_{2}\left(h_{\alpha}\right)\right\}=\lambda_{2}\left(h_{\alpha}\right)$. Which implies that if we define

$$
\mathbf{a} \in \mathbb{Z}^{n(n-1) / 2} \text { via } a_{\alpha}=\min \left\{\lambda_{1}\left(h_{\alpha}\right), \lambda_{2}\left(h_{\alpha}\right)\right\}
$$

then $a_{\alpha}=\lambda_{2}\left(h_{\alpha}\right)$. Let us denote $V\left(\lambda_{2}\right)^{a}$ the associated graded module obtained through the PBW filtration $U\left(\mathfrak{n}^{-}\right)$on the highest weight vector $v_{\lambda_{2}} \in V\left(\lambda_{2}\right)$ (see [FFL11a] for more details). This is a module for $S\left(\mathfrak{n}^{-}\right)$, the associated graded algebra of $U\left(\mathfrak{n}^{-}\right)$.

Proposition 6.1. If $\lambda \gg \lambda_{2}$, then

$$
S\left(\mathfrak{n}^{-}\right) / \mathcal{I}(\mathbf{a}) \cong V\left(\lambda_{2}\right)^{a} .
$$

Proof. This is nothing but [FFL11a, Theorem A].

We are ready to prove:

Theorem 6.1. If $\lambda_{1} \gg \lambda_{2}$, then

and

$$
a_{\lambda_{1}, \lambda_{2}}^{\tau}=c_{\lambda_{1}, \lambda_{2}}^{\tau}
$$

for all $c_{1} \neq c_{2} \in \mathbf{C}$.

$$
F_{\lambda_{1}, \lambda_{2}} \cong_{\mathfrak{s l}_{n} \otimes \mathbf{C}[t]} V\left(\lambda_{1}\right)_{c_{1}} * V\left(\lambda_{2}\right)_{c_{2}}
$$


Proof. With Corollary 4.2 we see that

$$
a_{\lambda_{1}, \lambda_{2}}^{\tau} \leq \sharp\left\{\mathbf{s} \in S\left(\lambda_{1}, \lambda_{2}\right) \mid \operatorname{wt}(\mathbf{s})=\lambda_{1}+\lambda_{2}-\tau\right\} .
$$

On the other hand, by Lemma 5.1, we have

$$
a_{\lambda_{1}, \lambda_{2}}^{\tau} \geq c_{\lambda_{1}, \lambda_{2}}^{\tau}
$$

By assumption $\lambda_{1} \gg \lambda_{2}$, which implies (Remark 3.1)

$$
c_{\lambda_{1}, \lambda_{2}}^{\tau}=\operatorname{dim} V\left(\lambda_{2}\right)_{\tau-\lambda_{1}} .
$$

Now [FFL11a, Theorem B] gives in this case a parametrization of a basis of $V\left(\lambda_{2}\right)$ in terms of (in our notation) $S\left(\lambda_{1}, \lambda_{2}\right)$, namely

$$
\operatorname{dim} V\left(\lambda_{2}\right)_{\tau-\lambda_{1}}=\sharp\left\{\mathbf{s} \in S\left(\lambda_{1}, \lambda_{2}\right) \mid \operatorname{wt}(\mathbf{s})=\lambda_{2}-\left(\tau-\lambda_{1}\right)\right\} .
$$

Which implies also

hence the equality follows.

$$
a_{\lambda_{1}, \lambda_{2}}^{\tau} \leq c_{\lambda_{1}, \lambda_{2}}^{\tau}
$$

\section{ReCtangular Weights}

In this section we prove generators and relations for the fusion product of two arbitrary Kirillov-Reshetikhin modules. These modules are defined in the context of simple, finitedimensional modules for the quantum affine algebra. They are indexed by a node $i \in I$, a level $m$ and an evaluation parameter $a \in \mathbf{C}(q)^{*}$ and denoted $K R\left(m \omega_{i}, a\right)$. For more on their importance we refer here to the survey [CH10].

In this paper we consider the non-quantum analog (obtained through the $q \mapsto 1$ limit). In the $\mathfrak{s l}_{n}$-case, they are isomorphic to evaluation modules $V\left(m \omega_{i}\right)_{c}$ for some $c \in \mathbf{C}$.

We have seen in Lemma 5.1 that

$$
F_{m_{i} \omega_{i}, m_{j} \omega_{j}} \rightarrow V\left(m_{i} \omega_{i}\right)_{c_{1}} * V\left(m_{j} \omega_{j}\right)_{c_{2}}
$$

for all $c_{1} \neq c_{2}$. We want to prove that this map is in fact an isomorphism, so we have to show that for all $\tau \in P^{+}$

$$
a_{m_{i} \omega_{i}, m_{j} \omega_{j}}^{\tau}=c_{m_{i} \omega_{i}, m_{j} \omega_{j}}^{\tau} .
$$

7.1. First, we will give formulas for the right hand side. We refer here to Nak93 where the decomposition of a tensor product was computed by using combinatorics of Young tableaux. A formula for the tensor product of $V\left(\lambda_{1}\right)$ with $V\left(\omega_{1}\right)$ is given explicitly and as well as the induction procedure for $V\left(\lambda_{2}\right)$. In the special case of $\lambda_{1}=m_{i} \omega_{i}$ and $\lambda_{2}=m_{j} \omega_{j}$ one can deduce straightforward that for all $\tau \in P^{+}$:

$$
c_{m_{i} \omega_{i}, m_{j} \omega_{j}}^{\tau} \in\{0,1\} .
$$

Moreover

Proposition 7.1. For $i \leq j, c_{m_{i} \omega_{i}, m_{j} \omega_{j}}^{\tau}=1$ if and only if (setting $\omega_{n}=\omega_{0}=0$.)

$$
\tau=m_{i} \omega_{i}+m_{j} \omega_{j}+\sum_{q \geq 0}^{\min \{i, j+i, n-j\}} b_{q}\left(\omega_{i-q}+\omega_{j+q}-\omega_{i}-\omega_{j}\right)
$$


with

$$
\sum_{q \geq 0}^{\min \{i, j+i, n-j\}} b_{q} \leq \min \left\{m_{i}, m_{j}\right\} \quad, b_{q} \geq 0
$$

7.2. Second, we will compute $a_{m_{i} \omega_{i}, m_{j} \omega_{j}}^{\tau}$. For this we identify again

$$
\mathbf{f}^{\mathbf{s}} \leftrightarrow \prod_{\alpha}\left(f_{\alpha} \otimes t\right)^{s_{\alpha}} .
$$

Recall, from Section 4 (and [FFL11a]) that $\mathfrak{n}^{+}$acts by differential operators on $S\left(\mathfrak{n}^{-}\right.$). Here, we introduce a new class of operators as follows. Let $R_{\lambda_{1}, \lambda_{2}}^{+}=\left\{\alpha \in R^{+} \mid \lambda_{1}\left(h_{\alpha}\right)=\lambda_{2}\left(h_{\alpha}\right)=0\right\}$. Then $\mathfrak{n}_{\lambda_{1}, \lambda_{2}}^{-}=\left\langle f_{\alpha} \mid \alpha \in R_{\lambda_{1}, \lambda_{2}}^{+}\right\rangle$is a subalgebra. We define for $\alpha \in R_{\lambda_{1}, \lambda_{2}}^{+}, \beta \in R^{+}$:

$$
f_{\alpha} \circ f_{\beta} \otimes t=\left\{\begin{array}{l}
f_{\alpha+\beta} \otimes t \text { if } \alpha+\beta \in R^{+} \\
0 \quad \text { else }
\end{array}\right.
$$

This is induced by the adjoint action of $\mathfrak{n}^{-}$on $\mathfrak{n}^{-} \otimes t$ (we normalize if necessary here). Moreover

Proposition 7.2. This action induces an action of differential operators on $U\left(\mathfrak{n}^{-} \otimes t\right)$. $\mathbb{1} \subset$ $F_{\lambda_{1}, \lambda_{2}}$.

Proof. This follows easily from the fact that $\mathfrak{n}_{\lambda_{1}, \lambda_{2}}^{-} \cdot \mathbb{1}=0 \in F_{\lambda_{1}, \lambda_{2}}$.

In the following we will abbreviate $f_{\alpha_{k, \ell}}$ with $f_{k, \ell}, s_{\alpha_{k, \ell}}$ with $s_{k, \ell}$. Denote further $\mathbf{e}_{k, l}$, the basis vector of $\mathbb{R}^{n(n-1) / 2}$ having 1 for $e_{\alpha_{k, \ell}}$ and 0 elsewhere. So let $\alpha \in R_{\lambda_{1}, \lambda_{2}}^{+}$and $\gamma=\alpha+\beta \in R^{+}$, then

$$
f_{\alpha} \circ \mathbf{f}^{\mathbf{e}_{\beta}}=\mathbf{f}^{\mathbf{e}_{\gamma}} .
$$

7.3. We turn to the case $\lambda_{1}=m_{i} \omega_{i}, \lambda_{2}=m_{j} \omega_{j}$. Let $\mathbf{s} \in S\left(\lambda_{1}, \lambda_{2}\right)$, then $s_{k, \ell}=0$ for $\ell<j$ or $k>i$. The following is the crucial lemma, which gives an upper bound for the set of highest weight points.

Lemma 7.1. Let $i \leq j \in I, m_{i}, m_{j} \geq 0$, and $p:=\min \{i-1, n-1-j\}$, then

$$
U\left(\mathfrak{n}^{-} \otimes t\right) . \mathbb{1} \subset U\left(\mathfrak{n}^{-}\right)\left\langle\left(f_{i, j} \otimes t\right)^{a_{0}}\left(f_{i-1, j+1} \otimes t\right)^{a_{1}} \cdots\left(f_{i-p, j+p}\right)^{a_{k}} \cdot \mathbb{1} \mid a_{q} \geq 0, \forall q\right\rangle .
$$

Moreover we have

$$
S_{h w}\left(\lambda_{1}, \lambda_{2}\right) \subseteq\left\{\mathbf{s} \in S\left(m_{i} \omega_{i}, m_{j} \omega_{j}\right) \mid s_{k, \ell}=0 \text { if }(k, \ell) \neq(i-q, j+q) \text { for some } q\right\} .
$$

Proof. We have seen in Corollary 4.1, that

$$
\left\{\mathbf{f}^{\mathbf{s}} . \mathbb{1} \mid \mathbf{s} \in S\left(\lambda_{1}, \lambda_{2}\right)\right\}
$$

generates $F_{\lambda_{1}, \lambda_{2}}$ as a $U\left(\mathfrak{n}^{-}\right)$-modules.

In our case $\lambda_{1}=m_{i} \omega_{i}, \lambda_{2}=m_{j} \omega_{j}$ and let $\mathbf{s} \in \mathbb{Z}_{\geq 0}^{n(n-1) / 2}$ with $s_{p, q}=0$ for $q<j$ or $p>i$. Let $k, \ell$ be such that $i-k>\ell-j, s_{k, \ell} \neq 0$ and

Condition (1) : $s_{r, \ell}=0, \forall r=1, \ldots, k-1$

Condition (2): $s_{r, s}=0$ if $r<k$ and $s<j+i-r$, then 
So $\mathbf{s}$ is of the form:

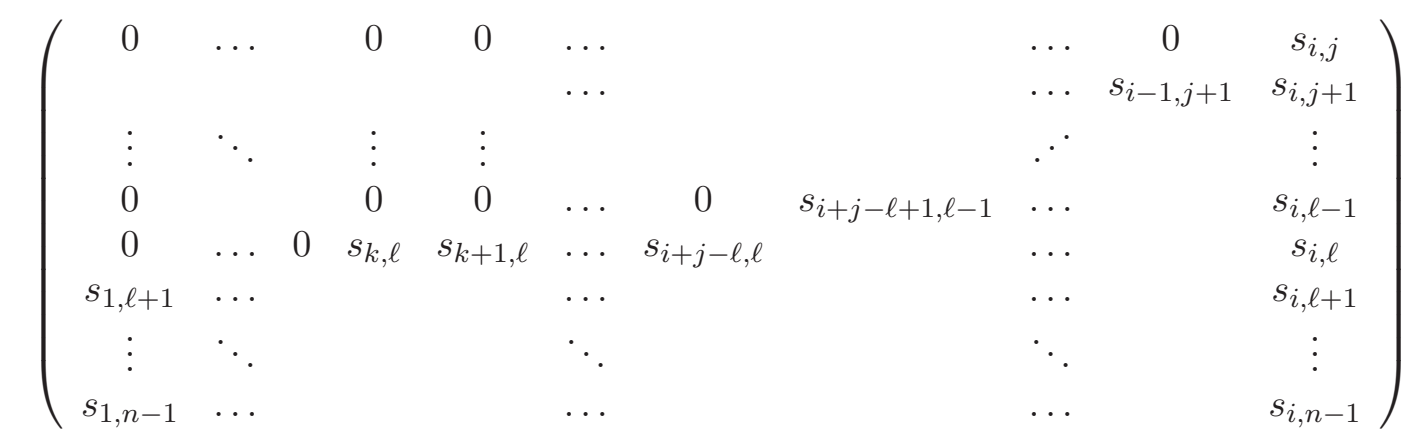

We consider

$$
f_{k, k} \circ\left(\mathbf{f}^{\mathbf{s}-\mathbf{e}_{k, \ell}+\mathbf{e}_{k+1, \ell}}\right) \cdot \mathbb{1}
$$

By expanding this we see that

$$
\left(s_{k+1, \ell}+1\right) \mathbf{f}^{\mathbf{S}} \cdot \mathbb{1}=\left[f_{k, k} \circ\left(\mathbf{f}^{\mathbf{S}-\mathbf{e}_{k, \ell}+\mathbf{e}_{k+1, \ell}}\right)-\sum_{z=\ell+1}^{n-1} s_{k+1, z}\left(\mathbf{f}^{\mathbf{s}-\mathbf{e}_{k, \ell}+\mathbf{e}_{k+1, \ell}+\mathbf{e}_{k, z}-\mathbf{e}_{k+1, z}}\right)\right] . \mathbb{1} .
$$

By iterating this we see that

$$
\mathbf{f}^{\mathbf{s}} \cdot \mathbb{1} \in \sum_{\mathbf{n}_{\alpha}} U\left(\mathfrak{n}^{-}\right) \mathbf{f}^{\mathbf{n}} \cdot \mathbb{1}
$$

where the sum is over all $\mathbf{n} \in \mathbb{Z}_{>0}^{n(n-1) / 2}$ satisfying Condition (1) and (2) and moreover $n_{k, \ell}=0$. Using induction along the first row, then along the second row etc, we see that

$$
\mathbf{f}^{\mathbf{s}} \cdot \mathbb{1} \in \sum_{\mathbf{n}} U\left(\mathfrak{n}^{-}\right) \mathbf{f}^{\mathbf{n}} \cdot \mathbb{1}
$$

where $n_{k, \ell}=0$ for all $k, \ell$ with $i-k>\ell-j$.

A similar computation for the roots below the diagonal shows that we can assume also $n_{k, \ell}=0$ for all $(k, \ell) \neq(i-q, j+q)$ for some $q$. This proves the first part of the lemma. The claim on highest weight points follows now from the definition of $F_{\lambda_{1}, \lambda_{2}}$, namely

$$
\left(f_{i-q, j+q} \otimes t\right)^{K} \cdot \mathbb{1}=0 \text { for } K \geq \min \left\{m_{i}, m_{j}\right\} .
$$

The following gives a stricter upper bound for the set of highest weight points.

Proposition 7.3. Let $i \leq j \in I, m_{i}, m_{j} \geq 0, p=\min \{i-1, n-1-j\}$, then $S_{h w}\left(\lambda_{1}, \lambda_{2}\right) \subseteq\left\{a_{0} \mathbf{e}_{i, j}+a_{1} \mathbf{e}_{i-1, j+1}+\ldots+a_{p} \mathbf{e}_{i-p, j+p} \mid \min \left\{m_{i}, m_{j}\right\} \geq a_{0} \geq a_{1} \geq \ldots \geq a_{p} \geq 0\right\}$.

Proof. We just have to check that these are the only points of the ones described in Lemma 7.1 whose monomials applied on $\mathbb{1}$ give vectors of dominant weight. For this, the weight of the vector

is equal to

$$
\left(f_{i, j} \otimes t\right)^{a_{0}}\left(f_{i-1, j+1} \otimes t\right)^{a_{1}} \cdots\left(f_{i-p, j+p}\right)^{a_{k}} \cdot \mathbb{1}
$$

$$
m_{i} \omega_{i}+m_{j} \omega_{j}+\sum_{q=0}^{p} a_{p}\left(\omega_{i-q}+\omega_{j+q}-\omega_{i-q-1}-\omega_{j+q+1}\right) .
$$


This is equal to

$$
\left(m_{i}-a_{0}\right) \omega_{i}+\left(a_{0}-a_{1}\right) \omega_{i-1}+\ldots+\left(a_{p-1}-a_{p}\right) \omega_{p}+\left(m_{j}-a_{0}\right) \omega_{j}+\ldots+\left(a_{p-1}-a_{p}\right) \omega_{j+p}
$$

which is dominant if and only if $a_{0} \geq a_{1} \geq \ldots \geq a_{p}$.

Keep the notation from the proof and set $b_{i}=a_{i}-a_{i+1} \geq 0$, then the weight of

$$
\left(f_{i, j} \otimes t\right)^{a_{0}}\left(f_{i-1, j+1} \otimes t\right)^{a_{1}} \cdots\left(f_{i-p, j+p}\right)^{a_{k}} \cdot \mathbb{1}
$$

is equal

$$
m_{i} \omega_{i}+m_{j} \omega_{j}+\sum_{q=0}^{\min \{i-1, n-1-j\}} b_{q}\left(\omega_{i-q}+\omega_{j+q}-\omega_{i}-\omega_{j}\right)
$$

with

$$
\sum_{q=0}^{\min \{i-1, n-1-j\}} b_{q}=a_{0} \leq \min \left\{m_{i}, m_{j}\right\}
$$

This implies

Theorem 7.1. Let $i, j \in I, m_{i}, m_{j} \geq 0$, then

$$
a_{m_{i} \omega_{i}, m_{j} \omega_{j}}^{\tau}=c_{m_{i} \omega_{i}, m_{j} \omega_{j}}^{\tau}
$$

for all $\tau \in P^{+}$and hence

$$
F_{m_{i} \omega_{i}, m_{j} \omega_{j}} \cong_{\mathfrak{s l}_{n} \otimes \mathbf{C}[t]} V\left(m_{i} \omega_{i}\right)_{c_{1}} * V\left(m_{j} \omega_{j}\right)_{c_{2}}
$$

for all $c_{1} \neq c_{2} \in \mathbf{C}$.

\section{The Pieri Rules}

In this section we want to compute the $\mathfrak{s l}_{n}$ decomposition on $F_{\lambda, \omega_{j}}$ and $F_{\lambda, k \omega_{1}}$. Mainly, we want to identify them with the fusion product of $V(\lambda)$ and $V\left(\omega_{j}\right)\left(\operatorname{resp} . V\left(k \omega_{1}\right)\right)$. As for the Kirillov-Reshetikhin modules we will show that $a_{\lambda, \omega_{j}}^{\tau}=c_{\lambda, \omega_{j}}^{\tau}$ for all $\tau$ and similar for $k \omega_{1}$. Let us start with the latter case.

On one hand, using again the Young tableaux combinatorics from [Nak93, we see that the highest weight vectors of $V(\lambda) \otimes V\left(k \omega_{1}\right)$ are parameterized by the set

$$
T_{\lambda, k \omega_{1}}:=\left\{\left(b_{1}, \ldots, b_{n}\right) \in \mathbb{Z}_{\geq 0}^{n} \mid, b_{1}+\ldots+b_{n}=k \text { and } b_{j} \leq m_{j-1} \forall j=2, \ldots, n\right\}
$$

where $\lambda=\sum m_{i} \omega_{i}$.

Let $\mathbf{s} \in S_{h w}\left(\lambda, k \omega_{1}\right) \subseteq S\left(\lambda, k \omega_{1}\right)$, then $s_{i, j}=0$ if $i \neq 1$. So there is no confusion if we write in the following $s_{j}$ for $s_{1, j}$. We have $s_{1}+\ldots+s_{n-1} \leq k$. Suppose now $s_{j}>m_{j}$ for some $j>1$, then by definition of $F_{\lambda, k \omega_{1}}$

$$
f_{j}^{s_{j}} \cdot \mathbb{1}=0
$$

This implies, recall the notation of Section 7 ,

$$
\mathbf{f}^{\mathbf{S}+s_{j} \mathbf{e}_{1, j-1}-s_{j} \mathbf{e}_{1, j}} f_{j}^{s_{j}} \cdot \mathbb{1}=0
$$


Using commutator relations we have for some constants $c_{k}$

$$
\sum_{k=0}^{s_{j}} c_{k} f_{j}^{k} \mathbf{f}^{\mathbf{s}+k\left(\mathbf{e}_{1, j-1}-\mathbf{e}_{1, j}\right)} \cdot \mathbb{1}=0
$$

This implies that

$$
\mathbf{f}^{\mathrm{s}} \cdot \mathbb{1} \in \sum_{\mathbf{n}} U\left(\mathfrak{n}^{-}\right) \mathbf{f}^{\mathbf{n}} \cdot \mathbb{1}
$$

for some $\mathbf{n}$ with $n_{\ell}=s_{\ell}$ for $\ell>j$ and $n_{j}<s_{j}$. But this is a contradiction to $\mathbf{s} \in S_{h w}\left(\lambda, k \omega_{1}\right)$. This implies that if $\mathbf{s} \in S_{h w}\left(\lambda, k \omega_{1}\right)$ we have

$$
s_{i, j}=0 \text { for } i \neq 1, s_{j} \leq m_{j}, s_{1}+\ldots+s_{n-1} \leq k .
$$

This implies $\left|S_{h w}\left(\lambda, k \omega_{1}\right)\right| \leq\left|T_{\lambda, k \omega_{1}}\right|$. Using now Lemma 5.1 we have equality here and so

Lemma 8.1. For $\lambda \in P^{+}, k \geq 0$, we have

$$
a_{\lambda, k \omega_{1}}^{\tau}=c_{\lambda, k \omega_{1}}^{\tau} \text { for all } \tau \in P^{+}
$$

and so for all $c_{1} \neq c_{2} \in \mathbf{C}$

$$
F_{\lambda, k \omega_{1}} \cong V(\lambda)_{c_{1}} * V\left(k \omega_{1}\right)_{c_{2}}
$$

8.1. We consider here the $\omega_{j}$-case. As before, using Young Tableaux combinatorics from Nak93], we have that the highest weight vectors of $V(\lambda) \otimes V\left(\omega_{j}\right)$ are parameterized by the set $\left(\lambda=\sum m_{i} \omega_{i}\right)$

$$
T_{\lambda, \omega_{j}}:=\left\{\left(b_{1}<\ldots<b_{j}\right) \mid, b_{i} \in\{1, \ldots, n\} \text { s.t.: } b_{i-1} \neq b_{i}-1 \Rightarrow m_{b_{i}-1} \neq 0\right\} .
$$

Let $\mathbf{s} \in S_{h w}\left(\lambda, \omega_{j}\right) \subseteq S\left(\lambda, \omega_{j}\right)$, then $s_{k, \ell}=0$ if $\ell>j$ or $k<j$. We have for all Dyck path p: $\beta_{1}+\ldots+\beta_{s} \leq 1$. This implies that $s_{\beta} \in\{0,1\}$ for all $\beta$ and even more, that the support of $\mathbf{s}_{\alpha}$ is of the form

$$
\left\{\alpha_{i_{1}, j_{1}}, \ldots, \alpha_{i_{\ell}, j_{\ell}} \mid i_{1}<i_{2} \ldots<i_{\ell} \leq j \leq j_{\ell}<\ldots<j_{1}\right\}
$$

Let us parametrize this set as follows. Let $\alpha_{i_{1}, j_{1}}, \ldots, \alpha_{i_{\ell}, j_{\ell}}$ be given from the set and denote

$$
\left\{p_{1}<\ldots<p_{j-\ell}:=\{1, \ldots, j\} \backslash\left\{i_{1}, \ldots, i_{\ell}\right\} .\right.
$$

Then we associate

$$
\alpha_{i_{1}, j_{1}}, \ldots, \alpha_{i_{\ell}, j_{\ell}} \leftrightarrow\left(p_{1}<p_{2}<\ldots<p_{j-\ell}<j_{\ell}+1<\ldots<j_{1}+1\right)
$$

This gives a one to one correspondence to $j$-tuples of strictly increasing integers smaller equals to $n$, hence parameterizes a basis of $V\left(\omega_{k}\right)$.

Since we are interested in the highest weight vectors, we can exclude these tuples corresponding to vectors in $F_{\lambda, \omega_{j}}$ of non-dominant weight. The weight of such a vector $\left(p_{1}<p_{2}<\ldots<p_{j}\right)$ is given by

$$
\lambda+\left(\omega_{j}-\left(-\omega_{p_{1}-1}+\omega_{p_{1}}-\omega_{p_{2}-1}+\omega_{p_{2}}-\ldots-\omega_{p_{j-1}-1}+\omega_{p_{j-1}} \omega_{p_{j}-1}+\omega_{p_{j}}\right) .\right.
$$

With a short calculation one sees that this is dominant if and only if $p_{i} \neq p_{i+1}-1 \Rightarrow m_{p_{i}}-1>0$. This implies that $a_{\lambda, \omega_{j}}^{\tau} \leq c_{\lambda, \omega_{j}}^{\tau}$ for all $\tau \in P^{+}$. Using Lemma 5.1 implies now equality for all $\tau$ which proves 
Lemma 8.2. For $\lambda \in P^{+}, j \in\{1, \ldots, n-1\}=I$, we have

$$
a_{\lambda, \omega_{j}}^{\tau}=c_{\lambda, \omega_{j}}^{\tau} \text { for all } \tau \in P^{+}
$$

and so for all $c_{1} \neq c_{2} \in \mathbf{C}$

$$
F_{\lambda, \omega_{j}} \cong V(\lambda)_{c_{1}} * V\left(\omega_{j}\right)_{c_{2}}
$$

\section{Partial order And Weyl modules}

In CFS14 a partial order on pairs of dominant weight has been introduced. Let us recall here briefly the construction. Fix $\lambda \in P^{+}$and consider the partitions of $\lambda$ with two parts

$$
P(\lambda, 2)=\left\{\left(\lambda_{1}, \lambda_{2}\right) \in P^{+} \times P^{+} \mid \lambda_{1}+\lambda_{2}=\lambda\right\} .
$$

By abuse of notation we denote by $P(\lambda, 2)$ the orbits of the natural $S_{2}$ action on $P(\lambda, 2)$. In CFS14, the following partial order has been introduced on $P(\lambda, 2)$ : Let $\boldsymbol{\lambda}=\left(\lambda_{1}, \lambda-\lambda_{1}\right), \boldsymbol{\mu}=$ $\left(\mu_{1}, \lambda-\mu_{1}\right) \in P(\lambda, 2)$, then

$$
\boldsymbol{\lambda} \preceq \boldsymbol{\mu}: \Leftrightarrow \forall \alpha \in R^{+}: \min \left\{\lambda_{1}\left(h_{\alpha}\right),\left(\lambda-\lambda_{1}\right)\left(h_{\alpha}\right)\right\} \leq \min \left\{\mu_{1}\left(h_{\alpha}\right),\left(\mu-\mu_{1}\right)\left(h_{\alpha}\right)\right\} .
$$

Certain properties of this poset were proved in [CFS14 (and [Fou14]), e.g. there exists a smallest element in $P(\lambda, 2)$, the orbit of $(\lambda, 0)$. It is less obvious that there exists also a unique maximal element: let $\lambda=\sum_{i=1}^{n-1} m_{i} \omega_{i}$, and let $\left\{1 \leq i_{1}<\ldots<i_{k}\right\}=I_{\text {odd }}$ be the indices such that $m_{i}$ is odd. Then $\boldsymbol{\lambda}^{\max }=\left(\lambda_{1}^{\max }, \lambda_{2}^{\max }\right)$ given by

$$
\lambda_{1}^{\max }=\sum_{j=1}^{k}\left(\left(m_{i_{j}}+(-1)^{j}\right) / 2\right) \omega_{i_{s}}+\sum_{i \in I \backslash I_{o d d}}\left(m_{i} / 2\right) \omega_{i}, \quad \lambda_{2}^{\max }=\lambda-\lambda_{1}^{\max },
$$

is the unique maximal orbit in $P(\lambda, 2)$, CFS14, Proposition 5.3].

It was further shown that the cover relation of $\preceq$ on $P(\lambda, 2)$ is determined by the Weyl group action [CFS14, Proposition 6.1].

9.1. We want to relate the partial order and the modules $F_{\lambda_{1}, \lambda_{2}}$. Namely, we want to prove the following lemma:

Lemma 9.1. Suppose $\left(\lambda_{1}, \lambda-\lambda_{1}\right) \preceq\left(\mu_{1}, \lambda-\mu_{1}\right) \in P(\lambda, 2)$, then there exists a canonical surjective map of $\mathfrak{s l}_{n} \otimes \mathbf{C}[t]$-modules

$$
F_{\mu_{1}, \mu-\mu_{1}} \rightarrow F_{\lambda_{1}, \lambda-\lambda_{1}} .
$$

Proof. We have to compare the defining relations only. So let $\alpha \in R^{+}$, then on both modules we have

$$
\left(f_{\alpha} \otimes 1\right)^{\lambda\left(h_{\alpha}\right)+1} \cdot \mathbb{1}=0
$$

and also the highest weight is in both cases $\lambda$. Let $M_{1}=\min \left\{\mu_{1}\left(h_{\alpha}\right), \lambda-\mu_{1}\left(h_{\alpha}\right)\right\}$ and $M_{2}=\min \left\{\lambda_{1}\left(h_{\alpha}\right), \lambda-\lambda_{1}\left(h_{\alpha}\right)\right\}$, then by assumption $M_{1} \geq M_{2}$. By the defining relations of $F_{\lambda_{1}, \lambda-\lambda_{1}}$ we have

so especially

$$
(f \otimes t)^{M_{2}+1} \cdot \mathbb{1}=0 \in F_{\lambda_{1}, \lambda-\lambda_{1}}
$$

$$
(f \otimes t)^{M_{1}+1} \cdot \mathbb{1}=0 \in F_{\lambda_{1}, \lambda-\lambda_{1}} .
$$

This implies the lemma. 
9.2. We turn to the unique maximal element in $P(\lambda, 2), \lambda^{\max }=\left(\lambda_{1}^{\max }, \lambda_{2}^{\max }\right)$. In fact we

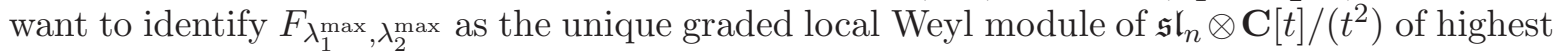
weight $\lambda$. For this we recall the definition of a local Weyl module briefly in the following.

Let $A$ be a commutative, finitely generated unital algebra over $\mathbf{C}$. Then $\mathfrak{s l}_{n} \otimes A$ is a Lie algebra with bracket given by

$$
[x \otimes p, y \otimes q]=[x, y] \otimes p q
$$

and it is called the generalized current algebra. We fix $\lambda \in P^{+}$, this induces an one-dimensional $\mathfrak{h}$-modules, which we denote $\mathbf{C}_{\lambda}$. Let $\xi:\left(\mathfrak{n}^{+} \oplus \mathfrak{h}\right) \otimes A \longrightarrow \mathfrak{h}$ be a Lie algebra homomorphism. Then we can lift the structure on $\mathbf{C}_{\lambda}$ to a $\left(\mathfrak{n}^{+} \oplus \mathfrak{h}\right) \otimes A$-structure, and let us denote this one-dimensional module $\mathbf{C}_{\lambda, \xi}$.

Definition 9.1. The local Weyl module $W_{A}(\xi, \lambda)$ is unique maximal integrable (as a $\mathfrak{s l}_{n^{-}}$ module) quotient of the $\mathfrak{s l}_{n} \otimes A$-module

$$
U\left(\mathfrak{s l}_{n} \otimes A\right) \otimes_{(\mathfrak{n}+\oplus \mathfrak{h}) \otimes A} \mathbf{C}_{\lambda, \xi}
$$

These modules have been introduced for $A=\mathbf{C}\left[t^{ \pm 1}\right]$ in [CP01] and further generalized in [FL04 and CFK10] to arbitrary commutative associative algebras over C. It has been shown in [CFK10] that if $A$ is finitely generated, $W_{A}(\xi, \lambda)$ is finite-dimensional and further that these modules are parameterized by maximal ideals in a tensor product of symmetric powers of $A$. These modules play an important role in the representation theory of $\mathfrak{s l}_{n} \otimes A$, the interested reader is here referred to [CFK10].

As they are integrable as $\mathfrak{s l}_{n}$-modules, there exist a decompositions into finite-dimensional simple $\mathfrak{s l}_{n}$-modules. Unfortunately, these decomposition are known for special cases only. Namely for $A=\mathbf{C}[t], \mathbf{C}\left[t^{ \pm 1}\right]$ they are computed in a series on paper [CP01, [CL06], [FL07]. If $A$ is semi-simple, then the local Weyl module obviously decomposes into a direct sum of local Weyl modules for $\mathfrak{s l}_{n} \otimes \mathbf{C}=\mathfrak{s l}_{n}$, so into a direct sum of simple $\mathfrak{s l}_{n}$-modules.

But outside of these cases, even for the "smallest" non-semi-simple algebra $A=\mathbf{C}[t] /\left(t^{2}\right)$, the $\mathfrak{s l}_{n}$ decomposition is unknown.

Let us rewrite the defining relations for the local Weyl modules for $A=\mathbf{C}[t] /\left(t^{K}\right)$. In fact, for each $\lambda \in P^{+}$and $K \geq 1$, there exists a unique local Weyl module. This follows since there exists a unique non-trivial map $\lambda \circ \xi$, namely $\xi$ is the evaluation map at $t=0$, so we denote $\xi$ by 0 .

Definition 9.2. Let $\lambda \in P^{+}$, then the graded local Weyl module $W_{\mathbf{C}[t] /\left(t^{K}\right)}(0, \lambda)$ is generated by $w \neq 0$ with relations

$$
\left(\mathfrak{n}^{+} \oplus \mathfrak{h}\right) \otimes t . w=0, h-\lambda(h) . w=0, \mathfrak{n}^{+} . w=0,\left(f_{\alpha} \otimes 1\right)^{\lambda\left(h_{\alpha}\right)+1} \cdot w=0 .
$$

Since the relations are homogeneous, we see that $W_{\mathbf{C}[t] /\left(t^{K}\right)}(0, \lambda)$ is a graded $\mathfrak{s l}_{n} \otimes \mathbf{C}[t] /\left(t^{K}\right)$ module. Even more, we have immediately from the defining relations

Proposition 9.1. Let $\lambda_{1}+\lambda_{2}=\lambda \in P^{+}$and $K \geq 2$, then there exists a surjective map of $\mathfrak{s l}_{n} \otimes \mathbf{C}[t]$-modules

$$
W_{\mathbf{C}[t] /\left(t^{K}\right)}(0, \lambda) \rightarrow F_{\lambda_{1}, \lambda_{2}} .
$$

In fact $F_{\lambda_{1}, \lambda_{2}}$ is the quotient obtained by factorizing the $U\left(\mathfrak{s l}_{n} \otimes \mathbf{C}[t]\right)$-submodule generated by

$$
\left\{\left(f_{\alpha} \otimes t\right)^{\min \left\{\lambda_{1}\left(h_{\alpha}\right), \lambda_{2}\left(h_{\alpha}\right)\right\}+1} . \mathbb{1} \mid \alpha \in R^{+}\right\} \cup\left\{f_{\alpha} \otimes t^{\ell} \mid \ell \geq 2, \alpha \in R^{+}\right\} .
$$


9.3. In this subsection we are restricting ourselves to the case of the second truncated current algebra, and we denote $A=\mathbf{C}[t] /\left(t^{2}\right)$. We will prove

Lemma 9.2. Let $\lambda \in P^{+}$and $\boldsymbol{\lambda}^{\max }=\left(\lambda_{1}^{\max }, \lambda_{2}^{\max }\right)$ be the unique maximal element in $P(\lambda, 2)$. Then we have an isomorphism of $\mathfrak{s l}_{n} \otimes A$-modules (and by extending an isomorphism of $\mathfrak{s l}_{n} \otimes \mathbf{C}[t]$-modules):

$$
W_{A}(0, \lambda) \cong F_{\lambda_{1}^{\max }, \lambda_{2}^{\max }}
$$

Proof. We consider the $\mathfrak{s l}_{2}$-case first. Then $\lambda=m \omega$ and because $e, e \otimes t, h \otimes t$ are acting trivial on $\mathbb{1}$,

$$
W_{A}(0, \lambda)=\operatorname{span}\left\{f^{K}(f \otimes t)^{L} \cdot w \mid, K, L \geq 0\right\}
$$

So if we restrict to elements in degree $L$ (recall, that $W_{A}(0, \lambda)$ is graded by the degree of $t$ ), then this is spanned by

$$
\left\{f^{K}(f \otimes t)^{L} \cdot w \mid K \geq 0\right\}
$$

The weights in degree $L$ are therefore of the form $m-2 L-2 K$ with $K \geq 0$. Every graded component is a finite-dimensional $\mathfrak{s l}_{2}$-module, since $\mathfrak{s l}_{2}$ acts by degree 0 and $W_{A}(0, \lambda)$ is finitedimensional. This implies that the component of degree $L$ in $W(0, \lambda)$ is 0 if there is no vector of dominant weight in degree $L$. So for $L>\lfloor m / 2\rfloor$ we have $(f \otimes t)^{L}$. w $=0$.

On the other hand, $\boldsymbol{\lambda}=(\lfloor m / 2\rfloor,\lceil m / 2\rceil)$ which implies that $F_{\lambda_{1}^{\max }, \lambda_{2}^{\max }}$ is the quotient by the submodule generated by

$$
(f \otimes t)^{L} . \mathbb{1} \text { with } L>\lfloor m / 2\rfloor .
$$

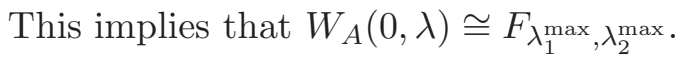

Let us turn to the general case. We have

$$
\min \left\{\lambda_{1}^{\max }\left(h_{\alpha}\right), \lambda_{2}^{\max }\left(h_{\alpha}\right)\right\}=\left\lfloor\lambda\left(h_{\alpha}\right) / 2\right\rfloor .
$$

It is enough to show that $\left(f_{\alpha} \otimes t\right)^{\left\lfloor\lambda\left(h_{\alpha}\right) / 2\right\rfloor+1} \cdot \mathbb{1}=0 \in W_{A}(0, \lambda)$ for all $\alpha$.

Fix $\alpha>0$ and consider the Lie subalgebra $\mathfrak{s l}(\alpha) \otimes A=\left\langle e_{\alpha}, h_{\alpha}, f_{\alpha}, e_{\alpha} \otimes t, h_{\alpha} \otimes t, f_{\alpha} \otimes t\right\rangle$ which is isomorphic to $\mathfrak{s l}_{2} \otimes A$.

We consider the submodule $M=U(\mathfrak{s l}(\alpha) \otimes A) \cdot \mathbb{1} \subseteq W_{A}(0, \lambda)$. Then this is a quotient of the $\mathfrak{s l}_{2} \otimes A$ local Weyl module $W_{A}\left(0, \lambda\left(h_{\alpha}\right) \omega\right)$ (since the defining relations are satisfied on the highest weight vector).

The considerations above for the $\mathfrak{s l}_{2}$-case imply now that

$$
\left(f_{\alpha} \otimes t\right)^{\left\lfloor\lambda\left(h_{\alpha}\right) / 2\right\rfloor+1} \cdot \mathbb{1}=0 \in M \subseteq W_{A}(0, \lambda)
$$

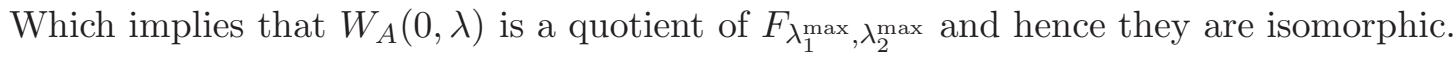

\section{REFERENCES}

[BD14] T. Backhaus and C. Desczyk. preprint, 2014.

[Bou02] Nicolas Bourbaki. Lie groups and Lie algebras. Chapters 4-6. Elements of Mathematics (Berlin). Springer-Verlag, Berlin, 2002. Translated from the 1968 French original by Andrew Pressley.

[CFK10] V. Chari, G. Fourier, and T. Khandai. A categorical approach to Weyl modules. Transform. Groups, 15(3):517-549, 2010.

[CFS14] V. Chari, G. Fourier, and D. Sagaki. Posets, Tensor products and Schur positivity. Algebra and Number theory, 2014.

[CH10] V. Chari and D. Hernandez. Beyond Kirillov-Reshetikhin modules. Quantum affine algebras, extended affine Lie algebras, and their applications, 506:49-81, 2010. 
[CL06] V. Chari and S. Loktev. Weyl, Demazure and fusion modules for the current algebra of $s l_{n+1}$. Adv.Math., 207(2):928-960, 2006.

[CM04] V. Chari and A. Moura. Spectral characters of finite-dimensional representations of affine algebras. J. Algebra, 279(2):820-839, 2004.

[CP01] V. Chari and A. Pressley. Weyl modules for classical and quantum affine algebras. Represent. Theory, 5:191-223 (electronic), 2001.

[CV13] V. Chari and R. Venkatesh. Demazure modules, fusion products and Q-systems. arXiv:1305.2523, 2013.

[DP07] G. Dobrovolska and P. Pylyavskyy. On products of $\mathfrak{s l}_{n}$ characters and support containment. J. Algebra, 316(2):706-714, 2007.

[ER93] S. Eswara Rao. On representations of loop algebras. Comm. Algebra, 21(6):2131-2153, 1993.

[FF02] B. Feigin and E. Feigin. Q-characters of the tensor products in $\mathfrak{s l}_{2}$-case. Mosc. Math. J., 2(3):567-588, 2002. Dedicated to Yuri I. Manin onthe occasion of his 65th birthday.

[FFL11a] E. Feigin, G. Fourier, and P. Littelmann. PBW filtration and bases for irreducible modules in type $\mathbf{A}_{n}$. Transform. Groups, 16(1):71-89, 2011.

[FFL11b] E. Feigin, G. Fourier, and P. Littelmann. PBW filtration and bases for symplectic Lie algebras. Int. Math. Res. Not. IMRN, 1(24):5760-5784, 2011.

[FFL13a] E. Feigin, G. Fourier, and P. Littelmann. Favourable modules: Filtrations, polytopes, NewtonOkounkov bodies and flat degenerations. arXiv:1306.1292v3, 2013.

[FFL13b] E. Feigin, G. Fourier, and P. Littelmann. Pbw-filtration over $\mathbb{Z}$ and compatible bases for $v(\lambda)$ in type $a_{n}$ and $c_{n}$. Springer Proceedings in Mathematics and Statistics, 40:35-63, 2013.

[FFLP05] S. Fomin, W. Fulton, C. Li, and Y. Poon. Eigenvalues, singular values, and Littlewood-Richardson coefficients. Amer. J. Math., 127(1):101-127, 2005.

[FL99] B. Feigin and S. Loktev. On generalized Kostka polynomials and the quantum Verlinde rule. Differentialtopology, infinite-dimensional Lie algebras, and applications, 194:61-79, 1999.

[FL04] B. Feigin and S. Loktev. Multi-dimensional Weyl modules and symmetric functions. Comm. Math. Phys., 251(3):427-445, 2004.

[FL06] G. Fourier and P. Littelmann. Tensor product structure of affine Demazure modules and limit constructions. Nagoya Math. J., 182:171-198, 2006.

[FL07] G. Fourier and P. Littelmann. Weyl modules, Demazure modules, KR-modules, crystals, fusion products and limit constructions. Adv. Math., 211(2):566-593, 2007.

[Fou14] G. Fourier. Extended partial order and applications to tensor products. Australasian Journal of Combinatorics 58 (1),178-196, (2014)., 58(1):178-196, 2014.

[Gor11] A. Gornitsky. Essential signatures and canonical bases in irreducible representations of the group $g_{2}$. Diploma thesis, 2011, 2011.

[Hag13] C. Hague. The induced PBW filtration, Frobenius splitting of double flag varieties, and Wahl's conjecture. arXiv:1309.0468, 2013.

[Kli68] A.U. Klimyk. Decomposition of a tensor product of irreducible representations of a semi-simple lie algebra into a direct sum of irreducible representations. Transl., II. Ser., Am. Math. Soc.76, 6373 (1968), 1968.

[Lit94] P. Littelmann. A Littlewood-Richardson rule for symmetrizable Kac-Moody algebras. Invent. Math., 116(1-3):329-346, 1994.

[LPP07] T. Lam, A. Postnikov, and P. Pylyavskyy. Schur positivity and Schur log-concavity. Amer. J.Math., 129(6):1611-1622, 2007.

[Nak93] T. Nakashima. Crystal base and a generalization of the Littlewood-Richardson rule for the classical Lie algebras. Comm. Math. Phys., 154(2):215-243, 1993.

[Nao12] K. Naoi. Weyl modules, Demazure modules and finite crystals for non-simply laced type. Adv. Math., 229(2):875-934, 2012.

[NSS12] E. Neher, A. Savage, and P. Senesi. Irreducible finite-dimensional representations of equivariant map algebras. Trans. Amer. Math. Soc., 364(5):2619-2646, 2012.

[Oko97] A. Okounkov. Log-concavity of multiplicities with application to characters of U( $\infty)$. Adv.Math., 127(2):258-282, 1997.

[Ven13] R. Venkatesh. Fusion product structure of Demazure modules. arXiv:1311.2224, 2013. 
Mathematisches Institut, Universität ZU Köln, Germany

E-mail address: gfourier@math.uni-koeln.de

School of Mathematics and Statistics, University of Glasgow, UK

E-mail address: ghislain.fourier@glasgow.ac.uk 